\title{
The impact of nonminimal universal extra dimensional model on $\Delta B=2$ transitions
}

\author{
Avirup Shaw ${ }^{\mathrm{a}}$ \\ Theoretical Physics, Physical Research Laboratory, Ahmedabad 380009, India
}

Received: 5 October 2020 / Accepted: 1 February 2021 / Published online: 8 February 2021

(C) The Author(s) 2021

\begin{abstract}
We measure the impact of nonvanishing boundary localised terms on $\Delta B=2$ transitions in fivedimensional Universal Extra Dimensional scenario where masses and coupling strengths of several interactions of Kaluza-Klein modes are significantly modified with respect to the minimal counterpart. In such scenario we estimate the Kaluza-Klein contributions of quarks, gauge bosons and charged Higgs by evaluating the one-loop box diagrams that are responsible for the $\Delta B=2$ transitions. Using the loop function (obtained from one-loop box diagrams) we determine several important elements that are involved in Wolfenstein parametrisation. Moreover, with these elements we also study the geometrical shape of unitarity triangle. Besides, we compute the quantity $\Delta M_{S}$ scaled by the corresponding Standard Model value. Outcomes of our theoretical predictions have been compared to the allowed ranges of the corresponding observables simultaneously. Our current analysis shows that, depending on the parameters in this scenario the lower limit on the inverse of the radius of compactification can reach to an appreciable large value $(\approx 1.48 \mathrm{TeV}$ or even higher).
\end{abstract}

\section{Introduction}

The Standard Model (SM) of particle physics has been a tremendously successful theory for explaining the features and interactions of fundamental particles, with many measurements confirming its predictions to extraordinary precision. With the discovery of the Higgs Boson by the ATLAS [1] and CMS [2] at the Large Hadron Collider (LHC) at CERN, all the particles in the SM zoo have been observed experimentally. However, it is known to be incomplete, as there exist several experimental data, such as massive neutrinos, Dark Matter (DM), matter anti-matter asymmetry etc., that cannot be explained in the SM scenario. Therefore, one of the current goals of particle physics is to discover new par-

a e-mail: avirup.cu@gmail.com (corresponding author) ticles and interactions-generically known as "new physics" (NP) that could provide an explanation for these observations. In principle, there are two ways to search for NP. At the high-energy frontier one tries to produce those new degrees of freedom directly, while at the high-precision frontier one analyses the indirect virtual effects of such new particles. In the second method NP would appear as a discrepancy between SM expectations and experimental measurements.

Considering the latter argument, we would like to mention that, one of the elegant ways to search for new particles is by studying processes known as flavour changing neutral current (FCNC) decays, where a quark changes its flavour without changing its electric charge. One example of such a transition is the decay of a bottom quark $(b)$ into a strange $(s)$ or down $(d)$ quark. In such classification, $B_{q^{-}}$ meson $(q=s, d)$ mixing is a particularly interesting process for indirect NP searches in the quark-flavour sector. Since in the SM such processes are forbidden at tree-level, they are sensitive to new heavy particles appearing as virtual particles in loop diagrams. Moreover, it is Glashow Iliopoulos Maiani (GIM) suppressed. The physical observables are the mass differences $\left(\Delta M_{q}\right)$, decay-width differences $\left(\Delta \Gamma_{q}\right)$ between the heavy and light neutral $B_{q}$-meson mass eigenstates, and the flavour-specific CP asymmetries $\left(a_{\mathrm{fs}}^{q}\right)$. Theoretical predictions of $B_{q}$-mixing observables in both the SM and beyond are governed by $\Delta B=2$ transitions and the hadronic matrix elements of which are expressed by local four-fermion operators in the effective weak Hamiltonian (given in Eq. 21). These observables are very useful to constrain physics beyond SM (BSM) scenarios. For example, in any BSM scenario if we compute the contribution of new heavy particles (particularly the contribution in box diagram for $B_{q}$-mixing) then it will contribute to loop-integral function ( Inami-Lim function [3]) of the corresponding operator which controls the $\Delta B=2$ transitions. Consequently, using the $B_{q}$-mixing observables we can measure the effects of that BSM scenarios by extracting the elements of the 
Cabibbo Kobayashi Maskawa (CKM) matrix [4,5] as well as by studying the shape of unitarity triangle (UT) [6]. Generally, four independent parameters are required to define the CKM matrix fully. Out of many parametrisations, Wolfenstein parametrisation [7] is the most famous and has several nice features. In particular it gives very prominent geometrical representation of the structure of the CKM matrix in conjunction with the UT which in turn very helpful to constrain the BSM scenarios. For example the Refs. [8-13] depicted how the observables are related to $\Delta B=2$ transition that can constrain different BSM scenarios. In the current article we consider a class of models, namely Universal Extra Dimensional (UED) [14] scenario, with nonvanishing boundary localised terms (BLTs), where the low energy effective Hamiltonians are controlled by local operators that are same as in the SM. In this type of scenario flavour violation and $\mathrm{CP}$ violation are entirely governed by the CKM matrix, with Minimal Flavour Violation (MFV) as defined in $[6,9,15,16]$.

UED [14] scenario is a specific extension of SM with one flat space-like dimension ( $y$ ) compactified on a circle $S^{1}$ of radius $R$. Each of the SM fields is exposed to the extra dimension $y$. The fields appeared on this manifold are generally defined as towers of 4-dimensional (4D) Kaluza-Klein (KK) states while the zero-mode of the KK-towers is recognised as the corresponding 4D SM field. SM chiral fermions are emerged in this scenario by imposing a discrete symmetry $Z_{2}(y \leftrightarrow-y)$ on the extra spatial dimension. Therefore, the extra dimension is known as an $S^{1} / Z_{2}$ orbifold and as a consequence physical domain extends from $y=0$ to $y=\pi R$. Eventually, the $y \leftrightarrow-y$ symmetry is appeared as a conserved parity which is designated as KK-parity $=(-1)^{n}$, where $n$ is known as KK-number and it measures the discretised momentum along the $y$-direction. Due to the conservation of KK-parity the lightest Kaluza-Klein particle (LKP) with KK-number one $(n=1)$ becomes absolutely stable and cannot decay to a pair of SM particles. Therefore, the LKP has been treated as a potential DM candidate in this scenario [17-24]. Besides, a few alternatives of this model can resolve some other demerits of SM, for example, gauge coupling unifications [25-27], neutrino mass [28,29] and fermion mass hierarchy [30] etc.

At the $n$th KK-level the mass of KK-partner of any SM particle can be expressed as $\sqrt{\left(m^{2}+\left(n R^{-1}\right)^{2}\right)}$, where $m$ is identified as the zero-mode mass (SM particle mass) and it is very small in comparison to $R^{-1}$. Eventually, this UED scenario consists of nearly degenerate mass spectrum at each KK-level. Due to this reason, UED scenario suffers from lack of phenomenological importance, particularly, at the colliders. However, radiative corrections [31,32] can cure the problem of degeneracy in the mass spectrum. The radiative corrections can be divided into two categories, e.g., the first one is bulk corrections (which are finite and only nonzero for $\mathrm{KK}$-excitations of gauge bosons) while the other one is considered as boundary localised corrections. The latter is proportional to logarithmically cut-off ${ }^{1}$ scale $(\Lambda)$ dependent terms. One can allow the boundary correction terms as 4D mass, kinetic and other possible interaction terms for the KK-excited states at the two fixed boundary points $(y=0$ and $y=\pi R$ ) of this orbifold. Actually, it is very natural to consider such terms in an extra dimensional theory like UED, because these boundary terms have served as the counterterms for cut-off dependent loop-induced contributions. There is a special assumption in the minimal version of UED (mUED) model, where the boundary terms are chosen in such a way that the $5 \mathrm{D}$ radiative corrections are disappeared at the cut-off scale $\Lambda$. Although, this unique assumption can be discarded and without computing the exact radiative corrections one could parametrise these as kinetic, mass as well as other interaction terms localised at the two fixed boundary points. Hence, this typical version is known as nonminimal Universal Extra Dimensional (nmUED) model [33-41]. Within this scenario, apart from the radius of compactification $(R)$, coefficients of different BLTs have been considered as free parameters and that can be constrained by various experimental data of several physical observables. In literature there exists number of phenomenological studies in this scenario. For example, bounds on the values of the coefficients of the BLTs have been obtained from the evaluation of electroweak observables $[39,41], \mathrm{S}, \mathrm{T}$ and $\mathrm{U}$ parameters $[37,42]$, DM relic density $[43,44]$, production as well as decay of SM Higgs boson [45], collider study of LHC experiments [4651], $Z \rightarrow b \bar{b}$ [52], branching ratios of some rare decay processes of $B$-meson: e.g., $B_{s} \rightarrow \mu^{+} \mu^{-}$[53], $B \rightarrow X_{s} \gamma$ [54] and $B \rightarrow X_{s} \ell^{+} \ell^{-}$[55], $\mathcal{R}_{D^{(*)}}$ anomalies [56-58], flavour changing rare top decay $[59,60]$ and unitarity of scattering amplitudes containing KK-excitations [61].

Within the scope of this article, to the best of our knowledge, for the first time we explore the $\Delta B=2$ transitions in the nmUED scenario by computing the KK-contributions to the one-loop box diagrams. The function emerging from the box diagrams is not only affected by the radius of compactification but also by the BLT parameters. Now it has already been mentioned that with the help of this function we can extract the CKM elements which in turn gives geometrical representation of the UT. Therefore, if we compare our theoretical prediction with the current allowed ranges of the CKM parameters and elements of the UT then we can easily constrain the parameter space of this nmUED framework. Moreover, from our study we can also measure the lower limit on $R^{-1}$ and compare the same with the results obtained from our previous analyses [53-55]. Similar kind of exercise was executed several years ago in UED framework [9] where the BLT parameters are zero. In the present article, consider-

\footnotetext{
${ }^{1}$ UED is regarded as an effective theory and it is characterised by a cut-off scale $\Lambda$.
} 
ing the current allowed ranges of the observables [62,63] we will also revisit the lower bound on $R^{-1}$ in UED framework with the BLT parameters as zero.

The paper is organised as follows. We will give a brief description of the nmUED model in Sect. 2. Then in Sect. 3 we will present the calculational details of $\Delta B=2$ transition in nmUED scenario. Consequently, we show the mechanism of the extraction of CKM parameters and the elements of UT. In Sect. 4 we will present our numerical results. Finally, we will summarise the results in Sect. 5 .

\section{A concise overview of KK-parity conserving nmUED scenario}

In this section we overview the salient features of the nmUED scenario necessary for our current analysis. One can find detailed description of this scenario in [33-40,46-56]. In order to conserve the KK-parity, coefficients of boundary terms at both the boundary points $(y=0$ and $y=\pi R$ ) are kept equal. Therefore, one has a stable LKP in this scenario and hence the present scenario can provide a potential DM candidate (such as first excited KK-state of the photon). In this type of scenario, one can find an extensive study on DM in [44].

Action for 5D fermionic fields considering appropriate boundary localised kinetic term (BLKT) with coefficient $r_{f}$ $[38,44,53-56]$ can be written as

$$
\begin{aligned}
S_{\text {fermion }}= & \int d^{5} x\left[\bar{\Psi}_{L} i \Gamma^{M} D_{M} \Psi_{L}\right. \\
& +r_{f}\{\delta(y)+\delta(y-\pi R)\} \bar{\Psi}_{L} i \gamma^{\mu} D_{\mu} P_{L} \Psi_{L} \\
& +\bar{\Psi}_{R} i \Gamma^{M} D_{M} \Psi_{R}+r_{f}\{\delta(y) \\
& \left.+\delta(y-\pi R)\} \bar{\Psi}_{R} i \gamma^{\mu} D_{\mu} P_{R} \Psi_{R}\right] .
\end{aligned}
$$

In the above action 5D four component Dirac spinors are represented by $\Psi_{L}(x, y)$ and $\Psi_{R}(x, y)$, which can be expressed in terms of two component spinors as [38,44,53-56]

$$
\begin{aligned}
& \Psi_{L}(x, y)=\left(\begin{array}{l}
\phi_{L}(x, y) \\
\chi_{L}(x, y)
\end{array}\right)=\sum_{n}\left(\begin{array}{l}
\phi_{L}^{(n)}(x) f_{L}^{n}(y) \\
\chi_{L}^{(n)}(x) g_{L}^{n}(y)
\end{array}\right), \\
& \Psi_{R}(x, y)=\left(\begin{array}{l}
\phi_{R}(x, y) \\
\chi_{R}(x, y)
\end{array}\right)=\sum_{n}\left(\begin{array}{l}
\phi_{R}^{(n)}(x) f_{R}^{n}(y) \\
\chi_{R}^{(n)}(x) g_{R}^{n}(y)
\end{array}\right) .
\end{aligned}
$$

Here, $f_{L(R)}$ and $g_{L(R)}$ are the KK-wave-functions that can be shown as the following form [34,39,44,53-56]

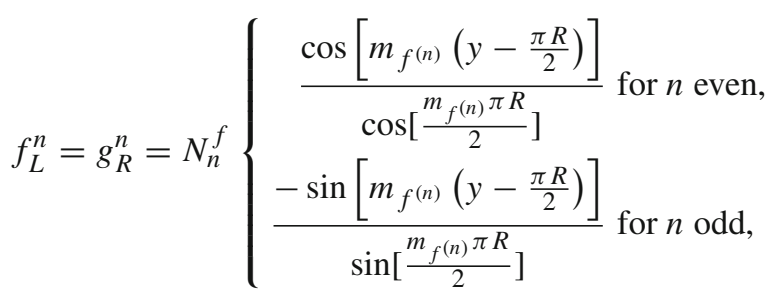

and

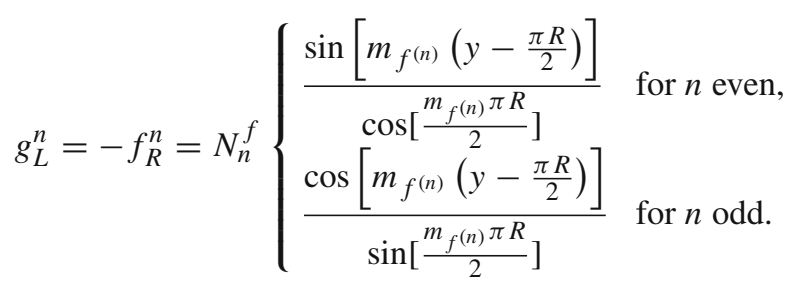

In the above expressions, $N_{n}^{f}$ represents the normalisation constant for $n$th KK-mode wave-function and can readily be derived from the following orthonormality conditions [44, 53-56]

$$
\left.\begin{array}{c}
\int_{0}^{\pi R} d y\left[1+r_{f}\{\delta(y)+\delta(y-\pi R)\}\right] f_{L}^{m} f_{L}^{n} \\
\int_{0}^{\pi R} d y\left[1+r_{f}\{\delta(y)+\delta(y-\pi R)\}\right] g_{R}^{m} g_{R}^{n}
\end{array}\right\}
$$

and the compact form of this normalisation constant is given by $[44,53-56]$

$N_{n}^{f}=\sqrt{\frac{2}{\pi R}}\left[\frac{1}{\sqrt{1+\frac{r_{f}^{2} m_{f^{(n)}}^{2}}{4}+\frac{r_{f}}{\pi R}}}\right]$.

KK-mass of $n$th KK-excitation is represented by $m_{f^{(n)}}$ and it can be obtained from the following transcendental equations [34,44,53-56]

$\frac{r_{f} m_{f^{(n)}}}{2}= \begin{cases}-\tan \left(\frac{m_{f^{(n)}} \pi R}{2}\right) & \text { for } n \text { even, } \\ \cot \left(\frac{m_{f^{(n)}} \pi R}{2}\right) & \text { for } n \text { odd. }\end{cases}$

To this end, we would like to discuss the Yukawa interactions in this scenario, as the large top quark mass plays a pivotal role in enhancing the quantum effects in the present work. The action of Yukawa interaction including BLTs with coefficient $r_{y}$ is given by [53-56]

$$
\begin{aligned}
S_{Y u k a w a}= & -\int d^{5} x\left[\lambda_{t}^{5} \bar{\Psi}_{L} \widetilde{\Phi} \Psi_{R}+r_{y}\{\delta(y)\right. \\
& \left.+\delta(y-\pi R)\} \lambda_{t}^{5} \overline{\phi_{L}} \widetilde{\Phi} \chi_{R}+\text { h.c. }\right] .
\end{aligned}
$$

In the above action $\lambda_{t}^{5}$ represents the $5 \mathrm{D}$ coupling strength of Yukawa interaction for the third generations. $\Phi=\left(\begin{array}{c}\phi^{+} \\ \phi^{0}\end{array}\right)$ is the 5D Higgs doublet field and $\widetilde{\Phi}=i \sigma^{2} \Phi^{*}$. Inserting the KK-wave-functions for fermions (given in Eqs. 2 and 3 ) in the actions given in Eqs. 1 and 9, one obtains the bilinear terms containing the doublet and singlet states of the quarks. The resulting mass matrix for the $n$th KK-level for 
third generation of quark can be written as the following [53-56]

$$
-\left({\overline{\phi_{L}}}^{(n)}{\overline{\phi_{R}}}^{(n)}\right)\left(\begin{array}{cc}
m_{f^{(n)} \delta^{n m}} & m_{t} \mathscr{I}_{1}^{n m} \\
m_{t} \mathscr{I}_{2}^{m n} & -m_{f^{(n)} \delta^{m n}}
\end{array}\right)\left(\begin{array}{l}
\chi_{L}^{(m)} \\
\chi_{R}^{(m)}
\end{array}\right)+\text { h.c. },
$$

where $m_{t}$ represents the mass of SM top quark and $m_{f^{(n)}}$ is derived from the solution of the transcendental equations given in Eq. 8. $\mathscr{I}_{1}^{n m}$ and $\mathscr{I}_{2}^{n m}$ are the overlap integrals that are given in the following [53-56]

$$
\begin{aligned}
\mathscr{I}_{1}^{n m}= & \left(\frac{1+\frac{r_{f}}{\pi R}}{1+\frac{r_{y}}{\pi R}}\right) \\
& \times \int_{0}^{\pi R} d y\left[1+r_{y}\{\delta(y)+\delta(y-\pi R)\}\right] g_{R}^{m} f_{L}^{n},
\end{aligned}
$$

and

$$
\mathscr{I}_{2}^{n m}=\left(\frac{1+\frac{r_{f}}{\pi R}}{1+\frac{r_{y}}{\pi R}}\right) \times \int_{0}^{\pi R} d y g_{L}^{m} f_{R}^{n} .
$$

The integral $\mathscr{I}_{1}^{n m}$ is nonvanishing for both the cases of $n=m$ and $n \neq m$. However, in the limit $r_{y}=r_{f}$, this integral becomes unity (when $n=m)$ or zero $(n \neq m)$. Besides, the integral $\mathscr{I}_{2}^{n m}$ is nonvanishing only for $n=m$ and becomes unity for $r_{y}=r_{f}$. At this point we would like to mention that, in our analysis in order to evade the complicacy of mode mixing and construct a simpler form of fermion mixing matrix we choose the condition of equality $\left(r_{y}=r_{f}\right)$ [52-56]. This equality condition ${ }^{2}\left(r_{y}=r_{f}\right)$ has been maintained in the rest of our analysis.

With the above mentioned equality condition $\left(r_{y}=r_{f}\right)$, the mass matrix (given in Eq. 10) can easily be diagonalised by the following bi-unitary transformations for the left- and right-handed fields [53-56]

$U_{L}^{(n)}=\left(\begin{array}{cc}\cos \alpha_{t n} & \sin \alpha_{t n} \\ -\sin \alpha_{t n} & \cos \alpha_{t n}\end{array}\right), \quad U_{R}^{(n)}=\left(\begin{array}{cc}\cos \alpha_{t n} & \sin \alpha_{t n} \\ \sin \alpha_{t n} & -\cos \alpha_{t n}\end{array}\right)$,

where, $\alpha_{t n}\left[=\frac{1}{2} \tan ^{-1}\left(\frac{m_{t}}{m_{f^{(n)}}}\right)\right]$ is identified as the mixing angle. The gauge eigen states $\Psi_{L}(x, y)$ and $\Psi_{R}(x, y)$ can be expressed in terms of mass eigen states $T_{t}^{1}$ and $T_{t}^{2}$ by the following relations [53-56]

$$
\begin{aligned}
& \phi_{L}^{(n)}=\cos \alpha_{t n} T_{t L}^{1(n)}-\sin \alpha_{t n} T_{t L}^{2(n)}, \\
& \phi_{R}^{(n)}=\sin \alpha_{t n} T_{t L}^{1(n)}+\cos \alpha_{t n} T_{t L}^{2(n)}, \\
& \chi_{L}^{(n)}=\cos \alpha_{t n} T_{t R}^{1(n)}+\sin \alpha_{t n} T_{t R}^{2(n)}, \\
& \chi_{R}^{(n)}=\sin \alpha_{t n} T_{t R}^{1(n)}-\cos \alpha_{t n} T_{t R}^{2(n)} .
\end{aligned}
$$

\footnotetext{
${ }^{2}$ However, in general, one can proceed with unequal coefficients of boundary terms for kinetic and Yukawa interaction for fermions.
}

Both the mass eigen states $T_{t}^{1(n)}$ and $T_{t}^{2(n)}$ have the identical mass eigen value at each KK-level. For $n$th KK-level the mass eigen value takes the form as $M_{t^{(n)}} \equiv \sqrt{m_{t}^{2}+m_{f^{(n)}}^{2}}$.

Let us look at the kinetic actions (governed by $S U(2)_{L} \times$ $U(1)_{Y}$ gauge group) of 5D gauge and scalar fields including their corresponding BLKTs $[39,52-56,59,64]$

$$
\begin{aligned}
S_{\text {gauge }}= & -\frac{1}{4} \int d^{5} x\left[W_{M N}^{a} W^{a M N}\right. \\
& +r_{W}\{\delta(y)+\delta(y-\pi R)\} W_{\mu \nu}^{a} W^{a \mu \nu} \\
& \left.+B_{M N} B^{M N}+r_{B}\{\delta(y)+\delta(y-\pi R)\} B_{\mu \nu} B^{\mu \nu}\right], \\
S_{\text {scalar }}= & \int d^{5} x\left[\left(D_{M} \Phi\right)^{\dagger}\left(D^{M} \Phi\right)\right. \\
& \left.+r_{\phi}\{\delta(y)+\delta(y-\pi R)\}\left(D_{\mu} \Phi\right)^{\dagger}\left(D^{\mu} \Phi\right)\right],
\end{aligned}
$$

where, $r_{W}, r_{B}$ and $r_{\phi}$ are designated as the coefficients of the BLKTs for the respective fields. 5D field strength tensors are expressed as

$W_{M N}^{a} \equiv\left(\partial_{M} W_{N}^{a}-\partial_{N} W_{M}^{a}-\tilde{g}_{2} \epsilon^{a b c} W_{M}^{b} W_{N}^{c}\right)$,

$B_{M N} \equiv\left(\partial_{M} B_{N}-\partial_{N} B_{M}\right)$.

$W_{M}^{a}\left(\equiv W_{\mu}^{a}, W_{4}^{a}\right)$ and $B_{M}\left(\equiv B_{\mu}, B_{4}\right)(M=0,1 \ldots 4)$ are considered as the $5 \mathrm{D}$ gauge fields corresponding to the gauge groups $S U(2)_{L}$ and $U(1)_{Y}$ respectively. 5D covariant derivative can be written as $D_{M} \equiv \partial_{M}+i \tilde{g}_{2} \frac{\sigma^{a}}{2} W_{M}^{a}+i \tilde{g}_{1} \frac{Y}{2} B_{M}$, where, $\tilde{g}_{2}$ and $\tilde{g}_{1}$ are represented as the $5 \mathrm{D}$ gauge coupling constants. Generators of $S U(2)_{L}$ and $U(1)_{Y}$ gauge groups are represented by $\frac{\sigma^{a}}{2}(a \equiv 1 \ldots 3)$ and $\frac{Y}{2}$ respectively. Each of the gauge and scalar fields which are involved in the above actions (Eqs. 13 and 14) can be manifested using appropriate KK-wave-functions as $[52-56,59,64]$

$$
V_{\mu}(x, y)=\sum_{n} V_{\mu}^{(n)}(x) a^{n}(y), \quad V_{4}(x, y)=\sum_{n} V_{4}^{(n)}(x) b^{n}(y)
$$

and

$\Phi(x, y)=\sum_{n} \Phi^{(n)}(x) h^{n}(y)$,

where both the 5D $S U(2)_{L}$ and $U(1)_{Y}$ gauge bosons are generically illustrated by $\left(V_{\mu}, V_{4}\right)$.

To this end, we would like to discuss some important points by which one can understand the following gauge and scalar field structure as well as the corresponding KKwave-functions. The physical neutral gauge bosons emerge from the mixing of $B$ and $W^{3}$ fields and hence the KKdecompositions of neutral gauge bosons are very complicated in the current extra dimensional scenario due to the presence of two types of mixings both at the bulk as well as on the boundary. Therefore, under this circumstances, it would be very difficult to diagonalise the bulk and boundary actions 
simultaneously by the same $5 \mathrm{D}$ field redefinition ${ }^{3}$ except the condition $r_{W}=r_{B}$. Hence, in the following, we will keep the equality condition $r_{W}=r_{B}[52-56,59,64]$ and as a consequence we obtain the same structure (like mUED scenario) of mixing between KK-excitations of the neutral component of the gauge fields (i.e., the mixing between $B^{(n)}$ and $\left.W^{3(n)}\right)$ in nmUED scenario. Thereafter, the mixing between $B^{(1)}$ and $W^{3(1)}$ (i.e., the mixing at the first KK-level) provides the $\gamma^{(1)}$ and $Z^{(1)}$. This $\gamma^{(1)}$ (first excited KK-state of the photon) is completely stable due to the conservation of KK-parity and it acquires the lowest mass among the first excited KK-states in the nmUED particle spectrum. Furthermore, it could not decay to pair of SM particles. Therefore, this $\gamma^{(1)}$ can be considered as a viable DM candidate in this scenario [44].

Let us discuss on the gauge fixing action (considering a generic BLKT parameter $r_{V}$ for gauge bosons) for nmUED scenario $[52-56,59,64]$

$$
\begin{aligned}
S_{\text {gauge fixing }}= & -\frac{1}{\xi_{y}} \int d^{5} x \mid \partial_{\mu} W^{\mu+}+\xi_{y}\left(\partial_{y} W^{4+}+i M_{W} \phi^{+}\{1\right. \\
& \left.\left.+r_{V}(\delta(y)+\delta(y-\pi R))\right\}\right)\left.\right|^{2} \\
& -\frac{1}{2 \xi_{y}} \int d^{5} x\left[\partial_{\mu} Z^{\mu}+\xi_{y}\left(\partial_{y} Z^{4}\right.\right. \\
& \left.\left.-M_{Z} \chi\left\{1+r_{V}(\delta(y)+\delta(y-\pi R))\right\}\right)\right]^{2} \\
& -\frac{1}{2 \xi_{y}} \int d^{5} x\left[\partial_{\mu} A^{\mu}+\xi_{y} \partial_{y} A^{4}\right]^{2},
\end{aligned}
$$

where $M_{W}\left(M_{Z}\right)$ is identified as the mass of the SM $W^{ \pm}(Z)$ boson. For an extensive study on gauge fixing action/mechanism in nmUED we refer to [64]. The above action (given in Eq. 18) is very intricate and at the same time very important for this nmUED scenario where we will compute one-loop diagrams (necessary for present calculation) in Feynman gauge. Due to the impact of the BLKTs, the Lagrangian leads to a non-homogeneous weight function for the fields with respect to the extra dimension. This inhomogeneity enforces us to define a $y$-dependent gauge fixing parameter $\xi_{y}$ as $[52-56,59,64]$

$\xi=\xi_{y}\left(1+r_{V}\{\delta(y)+\delta(y-\pi R)\}\right)$,

where $\xi$ is independent of $y$. The above relation behaves as renormalisation of the gauge fixing parameter since the BLKTs are in some sense contributed as the counterterms taking into account the unknown ultraviolet correction in loop calculations. With this in mind, we treat $\xi_{y}$ as the bare gauge fixing parameter while $\xi$ can be viewed as the renormalised gauge fixing parameter taking the values 0 (Landau gauge), 1 (Feynman gauge) or $\infty$ (Unitary gauge) [64].

\footnotetext{
${ }^{3}$ However, in general one can proceed with $r_{W} \neq r_{B}$, but in this case the mixing between $B$ and $W^{3}$ in the bulk and on the boundary points generate off-diagonal terms in the neutral gauge boson mass matrix.
}

In this nmUED scenario appropriate gauge fixing procedure enforces the equality condition of coefficient of the BLKTs of gauge and scalar fields, i.e., $r_{V}=r_{\phi}[52-$ $56,59,64]$. In this limit, KK-masses for the gauge and the scalar fields are equal $\left(m_{V^{(n)}}\left(=m_{\phi^{(n)}}\right)\right)$ and can be derived from the same transcendental equation (Eq. 8). At the $n$th KK-level the physical gauge fields $\left(W^{\mu(n) \pm}\right)$ and charged Higgs $\left(H^{(n) \pm}\right)$ have the same ${ }^{4}$ mass eigen value and is given by $[52-56,59,64]$

$$
M_{W^{(n)}}=\sqrt{M_{W}^{2}+m_{V^{(n)}}^{2}} .
$$

Besides, in the 't-Hooft Feynman gauge, the mass of Goldstone bosons $\left(G^{(n) \pm}\right)$ corresponding to the gauge fields $W^{\mu(n) \pm}$ have the same value $M_{W^{(n)}}[52-56,59,64]$.

To this end, we would like to focus on the interactions that will involve in our calculation. We can derive these interaction by integrating out the $5 \mathrm{D}$ action over the extra spacelike dimension $(y)$ using the specific $y$-dependent KK-wavefunction for the respective fields in 5D action. Consequently, some of the interactions are modified by so called overlap integrals with respect to their mUED counterparts. The actual form of the overlap integrals have been given in "Appendix A". Detailed discussions on these overlap integrals have been given in [53].

\section{$3 \Delta B=2$ transitions in nmUED scenario}

The effective Hamiltonian which governs the $\Delta B=2$ transitions in the SM $[65,66]$ can easily be modified for the nmUED scenario as follows

$$
\begin{aligned}
\mathcal{H}_{\mathrm{eff}}^{\Delta B=2}= & \frac{G_{\mathrm{F}}^{2}}{16 \pi^{2}} M_{W}^{2}\left(V_{t b}^{*} V_{t q}\right)^{2} \eta_{B} S\left(x_{t}, r_{f}, r_{V}, R^{-1}\right) \\
& \times\left[\alpha_{s}^{(5)}\left(\mu_{b}\right)\right]^{-6 / 23}\left[1+\frac{\alpha_{s}^{(5)}\left(\mu_{b}\right)}{4 \pi} J_{5}\right] \\
& \times\left[\bar{b} \gamma_{\mu}\left(1-\gamma_{5}\right) q\right]\left[\bar{b} \gamma^{\mu}\left(1-\gamma_{5}\right) q\right]+\text { h.c. },
\end{aligned}
$$

with $q=d, s$. Here $\mu_{b}=\mathcal{O}\left(m_{b}\right), J_{5}=1.627$ and

$\eta_{B}=0.55 \pm 0.01$

represents the short distance quantum chromo dynamics (QCD) corrections [65,66]. The function $S\left(x_{t}, r_{f}, r_{V}, R^{-1}\right)$ represents the total contribution in nmUED scenario as given below

\footnotetext{
${ }^{4}$ In the same way one can find the mass eigen values for the KK-excited $Z$ boson and pseudo scalar $A$. Also, their mass eigen values are identical to each other at any KK-level. For example at $n$th KK-level it takes the form as $\sqrt{M_{Z}^{2}+m_{V^{(n)}}^{2}}$.
} 


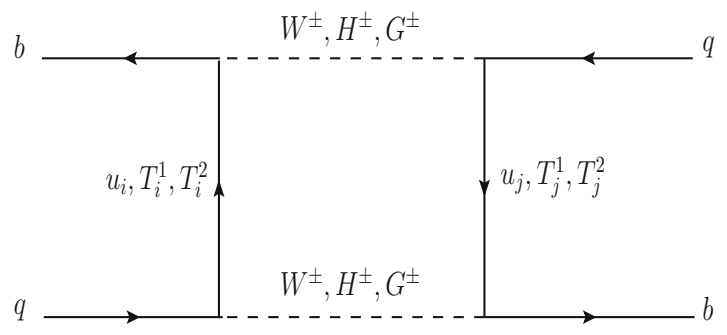

(a)

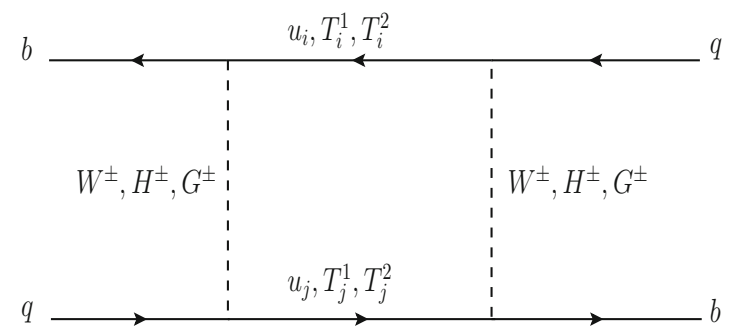

(b)

Fig. 1 The relevant box diagrams contributing to $S_{n}\left(x_{t}, x_{f^{(n)}}, x_{V^{(n)}}\right)$ in nmUED scenario

$S\left(x_{t}, r_{f}, r_{V}, R^{-1}\right)=S_{0}\left(x_{t}\right)+\sum_{n=1}^{\infty} S_{n}\left(x_{t}, x_{f^{(n)}}, x_{V^{(n)}}\right)$,

with $x_{t}=\frac{m_{t}^{2}}{M_{W}^{2}}, x_{V^{(n)}}=\frac{m_{V^{(n)}}^{2}}{M_{W}^{2}}$ and $x_{f^{(n)}}=\frac{m_{f^{(n)}}^{2}}{M_{W}^{2}} \cdot m_{V^{(n)}}$ and $m_{f^{(n)}}$ can be obtained from transcendental equation given in Eq. 8. Moreover,

$S_{0}\left(x_{t}\right)=\frac{4 x_{t}-11 x_{t}^{2}+x_{t}^{3}}{4\left(1-x_{t}\right)^{2}}-\frac{3 x_{t}^{3} \ln x_{t}}{2\left(1-x_{t}\right)^{3}}$

depicts the SM contribution $[9,67,68]$ and is obtained from the box diagrams with $\left(W^{ \pm}, t\right)$ and $\left(G^{ \pm}, t\right)$ exchanges with the $m_{t}$ independent terms eliminated by the GIM mechanism.

The function $S_{n}\left(x_{t}, x_{f^{(n)}}, x_{V^{(n)}}\right)$ indicate the KK-contributions which is obtained by evaluating the box diagrams shown in Fig. 1 with $W^{(n) \pm}, H^{(n) \pm}, G^{(n) \pm}, T_{i}^{1(n)}$ and $T_{i}^{2(n)}$ ( $i=u, c, t$ ) interchanges and multiplying the result by $i / 4$, where $1 / 4$ is a combinatorial factor. Momenta and masses of external quarks have been neglected in this calculation. Using the unitarity condition of CKM matrix we can write the functions $S_{n}\left(x_{t}, x_{f^{(n)}}, x_{V^{(n)}}\right)$ as [9]

$$
\begin{aligned}
S_{n}\left(x_{t}, x_{f^{(n)}}, x_{V^{(n)}}\right) \equiv & F\left(x_{t^{(n)}}, x_{t^{(n)}}\right)+F\left(x_{u^{(n)}}, x_{u^{(n)}}\right) \\
& -2 F\left(x_{t^{(n)}}, x_{u^{(n)}}\right),
\end{aligned}
$$

where the function $F\left(x_{i^{(n)}}, x_{j^{(n)}}\right)$ is representing the sum of the contribution of the diagrams corresponding to a given pair $\left(m_{i(n)}, m_{j(n)}\right)$ to $S_{n}\left(x_{t}, x_{f^{(n)}}, x_{V^{(n)}}\right)$, where

$x_{i(n)}=\frac{m_{i(n)}^{2}}{M_{W^{(n)}}^{2}}=\frac{m_{i}^{2}+m_{f^{(n)}}^{2}}{M_{W}^{2}+m_{V^{(n)}}^{2}}$.

Finally the compact form of this function obtained from oneloop box diagrams (see Fig. 1) is given by

$$
\begin{aligned}
& S_{n}\left(x_{t}, x_{f^{(n)}}, x_{V^{(n)}}\right) \\
& =\frac{1}{4}\left[\frac { 1 } { ( - 1 + x _ { f ^ { ( n ) } } - x _ { V ^ { ( n ) } } ) ^ { 3 } } \left[\left(-1+x_{f^{(n)}}-x_{V^{(n)}}\right)\right.\right. \\
& \quad \times\left\{( I _ { 2 } ^ { n } ) ^ { 4 } \left(1+x_{f^{(n)}}\right.\right.
\end{aligned}
$$

$$
\begin{aligned}
& \left.\left.-15 x_{V^{(n)}}\right)+4\left(I_{1}^{n}\right)^{4}\left(1+x_{f^{(n)}}+x_{V^{(n)}}\right)\right\} \\
& -2\left\{4\left(I_{1}^{n}\right)^{4} x_{f^{(n)}}\left(1+x_{V^{(n)}}\right)\right. \\
& \left.+\left(I_{2}^{n}\right)^{4}\left(x_{f^{(n)}}-3 x_{f^{(n)}} x_{V^{(n)}}-4 x_{V^{(n)}}\left(1+x_{V^{(n)}}\right)\right)\right\} \\
& \left.\times \ln \left(\frac{x_{f^{(n)}}}{1+x_{V^{(n)}}}\right)\right] \\
& -2\left[\frac{\left\{\left(I_{2}^{n}\right)^{4}\left(1-7 x_{V^{(n)}}\right)+4\left(I_{1}^{n}\right)^{4}\left(1+x_{V^{(n)}}\right)\right\}}{\left(-1+x_{f^{(n)}}-x_{V^{(n)}}\right)\left(-1+x_{t}+x_{f^{(n)}}-x_{V^{(n)}}\right)}\right. \\
& -\frac{x_{f^{(n)}}\left\{4\left(I_{1}^{n}\right)^{4} x_{f^{(n)}}+\left(I_{2}^{n}\right)^{4}\left(x_{f^{(n)}}-8 x_{V^{(n)}}\right)\right\}}{x_{t}\left(-1+x_{f^{(n)}}-x_{V^{(n)}}\right)^{2}} \ln \left(\frac{x_{f^{(n)}}}{1+x_{V^{(n)}}}\right) \\
& +\frac{\left(1+\frac{x_{f^{(n)}}}{x_{t}}\right)\left\{4\left(I_{1}^{n}\right)^{4}\left(x_{t}+x_{f^{(n)}}\right)+\left(I_{2}^{n}\right)^{4}\left(x_{t}+x_{f^{(n)}}-8 x_{V^{(n)}}\right)\right\}}{\left(-1+x_{t}+x_{f^{(n)}}-x_{V^{(n)}}\right)^{2}} \\
& \left.\times \ln \left(\frac{x_{f^{(n)}}+x_{t}}{1+x_{V^{(n)}}}\right)\right] \\
& +\frac{1}{\left(-1+x_{t}+x_{f^{(n)}}-x_{V^{(n)}}\right)^{3}}\left[\left(-1+x_{t}+x_{f^{(n)}}-x_{V^{(n)}}\right)\right. \\
& \times\left\{\left(I_{2}^{n}\right)^{4}\left(1+x_{t}+x_{f^{(n)}}-15 x_{V^{(n)}}\right)+\left(I_{1}^{n}\right)^{4}\left(x_{f^{(n)}}\left(4+x_{t}^{2}\right)\right.\right. \\
& \left.\left.+4\left(1+x_{V^{(n)}}\right)+x_{t}\left(4+x_{t}\left(-15+x_{t}+x_{V^{(n)}}\right)\right)\right)\right\} \\
& -2\left\{\left(I_{2}^{n}\right)^{4}\left(x_{f^{(n)}}+x_{t}-4 x_{V^{(n)}}-3\left(x_{f^{(n)}}+x_{t}\right) x_{V^{(n)}}-4 x_{V^{(n)}}^{2}\right)\right. \\
& +\left(I_{1}^{n}\right)^{4}\left(x_{t}\left(4-4 x_{t}-3 x_{t}^{2}+\left(x_{t}-2\right)^{2} x_{V^{(n)}}\right)\right. \\
& \left.\left.+x_{f^{(n)}}\left(x_{t}^{2}\left(-3+x_{V^{(n)}}\right)+4\left(1+x_{V^{(n)}}\right)\right)\right)\right\} \\
& \left.\left.\times \ln \left(\frac{x_{f^{(n)}}+x_{t}}{1+x_{V^{(n)}}}\right)\right]\right] .
\end{aligned}
$$

With the increasing value of KK-modes (i.e., with higher value $n$ ) the masses of the fields $T_{t}^{1(n)}, T_{t}^{2(n)}, T_{u}^{1(n)}$ and $T_{u}^{2(n)}$ become degenerate in nature and consequently the function $S_{n}\left(x_{t}, x_{f^{(n)}}, x_{V^{(n)}}\right)$ diminishes with larger values of $n$. Therefore, only a few terms in the sum given in Eq. 23 are relevant. Here, $I_{1}^{n}$ and $I_{2}^{n}$ are two overlap integrals and their expressions are given in the "Appendix A". 
It has already been addressed that, due to the presence of different BLTs in the nmUED action, the KK-masses and couplings (modified by $I_{1}^{n}$ and $I_{2}^{n}$ ) involving KK-excitations are nontrivially modified with respect to their UED counterparts. Therefore, it would not be possible to evaluate the function $S_{n}$ in nmUED scenario simply by rescaling the same of the UED model [9]. Hence, we have computed the function $S_{n}$ independently using the box diagram (Fig. 1) for the nmUED scenario. Moreover, it is quite clear from Eq. 27 that the function $S_{n}$ is drastically different from that of the UED expression. However, if we set the boundary terms to zero; i.e., $r_{V}=0$ and $r_{f}=0$, then we can easily reproduce the result of the UED version from our expression. Further, we would like to mention that in our computation of oneloop box diagrams we consider only those interactions in which zero-mode field couples to a pair of KK-excitations with equal KK-numbers. Moreover, in the KK-parity conserving nmUED scenario one can also have nonzero interactions containing KK-excitations with KK-numbers $n, m$ and $p$, where $n+m+p$ is an even integer. However, we have explicitly verified that the final results would not change significantly even if one considers the contributions of all the possible off-diagonal interactions [52-55].

To this end, applying the same procedure as in the SM, we can calculate the mass differences $\Delta M_{q}$ for nmUED scenario. For this purpose, we can readily adopt the technique of UED scenario as given in [9], because the basic structures (also the structure of operator responsible for $B_{q^{-}}$ mixing) of both UED and nmUED scenarios are similar. Therefore, all other physical aspects (apart from the function $S_{n}$ obtained from the one-loop box diagrams) of both the UED and nmUED scenarios are same. Hence, one can easily write the expression of mass difference in nmUED scenario as

$\Delta M_{q}=\frac{G_{\mathrm{F}}^{2}}{6 \pi^{2}} \eta_{B} m_{B_{q}}\left(\hat{B}_{B_{q}} F_{B_{q}}^{2}\right) M_{W}^{2} S\left(x_{t}, r_{f}, r_{V}, R^{-1}\right)\left|V_{t q}\right|^{2}$

Here, $F_{B_{q}}$ represents the $B_{q}$-meson decay constant and $\hat{B}_{q}$ the renormalisation group invariant parameter related to the hadronic matrix element of the operator $\Delta B=2$ [69].

At this moment, we would like to make a few comments on the QCD factor $\eta_{B}$ (given in Eq. 22) which has been evaluated within the SM including next-to-leading oder (NLO) QCD corrections. These are necessary for the proper matching of the Wilson Coefficient (WC) of the operator $(\Delta B=2)$ with its hadronic matrix element designated by the parameter $\hat{B}_{B_{s, d}}$ and evaluated using non-perturbative methods. Since the KK-modes and the top quark are integrated out at a single scale $\mu_{t}=\mathcal{O}\left(m_{\mathrm{t}}, R^{-1}\right)$, therefore at a scale lower than $\mu_{t}$, the contributions to $\eta_{B}$ for the nmUED scenario and for the SM are same. They just represent the finite renormalisation of the operator $(\Delta B=2)$ from the scales $\mathcal{O}\left(\mu_{t}\right)$

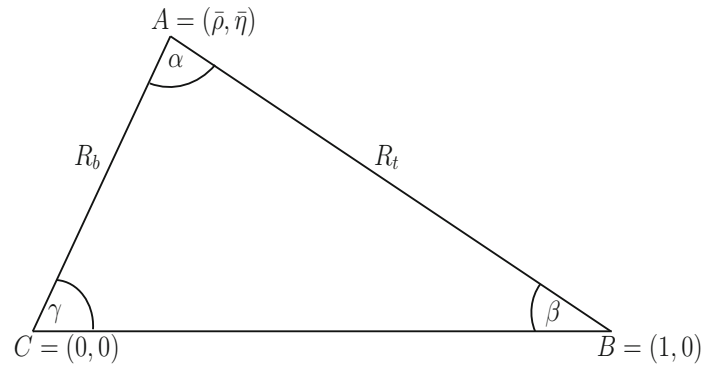

Fig. 2 Unitarity triangle

down to the scales $\mathcal{O}\left(m_{b}\right)$. The disagreement in QCD corrections between the KK-contributions and the SM contributions appears only in the full theory at scales $\mu_{t}=\mathcal{O}\left(m_{\mathrm{t}}, R^{-1}\right)$. In this situation the unknown QCD corrections to the box diagrams in Fig. 1 can, in principle, differ from the known QCD corrections to the SM box diagrams $[65,66]$ that have been included in $\eta_{B}$. However, as the QCD coupling constant $\alpha_{s}\left(\mu_{t}\right)$ is small and the QCD corrections to the SM box diagrams are of order of a few percent, therefore one can expect that the difference between the QCD corrections to the diagrams in Fig. 1 and to the SM box diagrams is insignificant [9]. Therefore, in the following, we will use the same QCD factor and hadronic matrix element in SM as well as in the concerned nmUED scenario. With this we will study the $\Delta B=2$ transitions in nmUED scenario and try to estimate the impact of BLT parameters on the CKM elements and UT.

\subsection{Effects of BLTs on CKM parameters and unitarity triangle in the nmUED scenario}

In nmUED scenario, we evaluate the KK-contributions to the function $S_{n}$ which is significantly affected by BLT parameters $\left(r_{V}, r_{f}\right)$. Therefore, using this function $S_{n}$ we can study the effects of BLT parameters on the elements of the CKM matrix and in particular on the shape of the UT. However, in order to execute this strategy we need to recall some features of CKM matrix and the UT as depicted in Fig. 2. Using the Wolfenstein parametrisation [7] as generalised to higher orders $^{5}$ in $\lambda$, the CKM matrix can be written as [67]

$\mathrm{V}_{\mathrm{CKM}}=\left(\begin{array}{ccc}1-\frac{\lambda^{2}}{2} & \lambda & \mathbb{A} \lambda^{3}(\rho-i \eta) \\ -\lambda & 1-\frac{\lambda^{2}}{2} & \mathbb{A} \lambda^{2} \\ \mathbb{A} \lambda^{3}\left[1-(\rho+i \eta)\left(1-\frac{\lambda^{2}}{2}\right)\right] & -\mathbb{A} \lambda^{2} & 1\end{array}\right)$.

\footnotetext{
5 One should note that here, terms greater than $\mathcal{O}\left(\lambda^{4}\right)$ have been neglected. However, this may give wrong results. In order to improve the accuracy of the UT a correction term $\approx \mathcal{O}\left(\lambda^{5}\right)$ has been included to $V_{t d}$ element.
} 
Here $\lambda, \mathbb{A}, \rho$ and $\eta$ are the Wolfenstein parameters [7]. The most commonly used UT arises from

$V_{u d} V_{u b}^{*}+V_{c d} V_{c b}^{*}+V_{t d} V_{t b}^{*}=0$,

by dividing each side by the best known one, $V_{c d} V_{c b}^{*}$. Its vertices are exactly $C=(0,0), B=(1,0)$, and $A=(\bar{\rho}, \bar{\eta})$, where $\bar{\rho}$ and $\bar{\eta}$ of the UT is given by [67]

$\bar{\rho}=\rho\left(1-\frac{\lambda^{2}}{2}\right), \quad \bar{\eta}=\eta\left(1-\frac{\lambda^{2}}{2}\right)$.

The lengths $R_{b}$ and $R_{t}$ can be expressed as $[9,67]$

$$
\begin{aligned}
& R_{b} \equiv \frac{\left|V_{u d} V_{u b}^{*}\right|}{\left|V_{c d} V_{c b}^{*}\right|}=\sqrt{\bar{\rho}^{2}+\bar{\eta}^{2}}=\left(1-\frac{\lambda^{2}}{2}\right) \frac{1}{\lambda}\left|\frac{V_{u b}}{V_{c b}}\right|, \\
& R_{t} \equiv \frac{\left|V_{t d} V_{t b}^{*}\right|}{\left|V_{c d} V_{c b}^{*}\right|}=\sqrt{(1-\bar{\rho})^{2}+\bar{\eta}^{2}}=\frac{1}{\lambda}\left|\frac{V_{t d}}{V_{c b}}\right|,
\end{aligned}
$$

while the angles $\gamma$ and $\beta$ of the UT are connected directly to the complex phases of the CKM elements $V_{t d}$ and $V_{u b}$, respectively, via

$V_{u b}=\left|V_{u b}\right| e^{-i \gamma}, V_{t d}=\left|V_{t d}\right| e^{-i \beta}$.

The value of $R_{b}$ (given in 32), i.e., the length of the side $\mathrm{AC}$ is determined from $\left|V_{u b} / V_{c b}\right| \cdot\left|V_{c b}\right|$ and $\left|V_{u b}\right|$ are in general determined from tree level decays. Moreover, in the nmUED scenario there are no KK-contributions at the tree level, hence absolute values of these CKM elements of nmUED scenario are approximately same as that of the SM. Besides, if we look from the perspective of the UT, the lengths of its two sides, $\mathrm{AC}$ and $\mathrm{CB}$ are common to the SM and the nmUED scenario. Moreover, we would like to mention that in the nmUED scenario as there are no new complex phases beyond the KM phase, the angle $\beta$ as extracted by means of $a_{\psi K_{S}}$ in $B \rightarrow \psi K_{S}$ is common to both nmUED scenario and SM. The world average value of the angle $\beta$ which we will use in our following analysis is given below [70]

$\beta=(22.2 \pm 0.7)^{\circ}$.

Before proceeding further, we would like to mention that, in view of the previous discussions we can easily categorise the present nmUED scenario as an MFV model $[15,16]$. The reason is that this nmUED scenario (like UED scenario [9]) is a class of extensions of the SM in which only the SM operators in the effective weak Hamiltonian are relevant and flavour violation is entirely governed by the CKM matrix. Moreover, $\mathrm{CP}$ violation is governed solely by the $\mathrm{KM}$ phase. Besides, one of the fascinating features of the MFV models is the existence of the universal UT (UUT) [6] that can be constructed from quantities in which all the dependence on NP cancels out or is negligible like in tree level decays. Now, in spite of the existence of common UUT for both the nmUED and SM scenarios, a crucial difference is
$S\left(x_{t}, r_{f}, r_{V}, R^{-1}\right) \neq S_{0}\left(x_{t}\right)$. Therefore, only one from the following observables: $\varepsilon_{K},{ }^{6} \Delta M_{d}$ and $\Delta M_{s}$ will agree with the experimental data for only one of the two scenarios (SM and nmUED).

At this stage, considering the above mentioned facts, we can present a picture which shows how the quantities $\left|V_{t d}\right|, \bar{\rho}$, $\bar{\eta}$ and $\gamma$ are affected by the BLT parameters $\left(r_{V}, r_{f}\right)$ and $R^{-1}$. In order to obtain these results in nmUED scenario we adopt the following procedure which is quite similar as given in [9] albeit for UED scenario. At first we use the $B_{d}^{0}-\bar{B}_{d}^{0}$ mixing constraint $\Delta M_{d}$. Using the central value of experimental data of $\Delta M_{d}=(0.5065 \pm 0.0019) \mathrm{ps}^{-1}[62,70]$ and utilising the function given in Eq. 23 we can determine ${ }^{7}$ the value of $\left|V_{t d}\right|$ in nmUED scenario from Eq. 28. After that, using this $\left|V_{t d}\right|$ and with the allowed value of $\lambda=0.224837_{-0.000060}^{+0.000251}$ [63] and $V_{c b}=(41.0 \pm 1.4) \times 10^{-3}$ [62] we will find $R_{t}$ via Eq. 33. Finally, using the following relations [15]

$$
\begin{aligned}
\bar{\eta} & =R_{t} \sin (\beta), \quad \bar{\rho}=1-R_{t} \cos (\beta) \quad \text { and } \\
\cot \gamma & =\frac{1-R_{t} \cos (\beta)}{R_{t} \sin (\beta)},
\end{aligned}
$$

we will compute the values of $\bar{\rho}, \bar{\eta}$ and $\gamma$ respectively, as functions of $R^{-1}$ and BLT parameters $\left(r_{V}, r_{f}\right)$ in nmUED scenario. With this procedure we can obtain the parameter space which satisfies $2 \sigma$ allowed range of the values of the quantities $\left(\left|V_{t d}\right|=(8.0 \pm 0.6) \times 10^{-3}[62], \bar{\rho}=0.157_{-0.012}^{+0.027}[63]\right.$, $\bar{\eta}=0.350_{-0.016}^{+0.018}[63]$ and $\left.\gamma=(72.1 \pm 8.6)^{\circ}[62]\right)$. As, for $\bar{\rho}$ and $\bar{\eta}$, the distributions are perfectly Gaussian [63], therefore, we can definitely take the values with $2 \sigma$ uncertainties for $\bar{\rho}$ and $\bar{\eta}$ with all other observables for which $2 \sigma$ uncertainties are given as twice the $1 \sigma$ uncertainty.

Additionally, with the above mentioned conditions we would also like to impose another condition simultaneously by studying the effects of BLT parameters $\left(r_{V}, r_{f}\right)$ and $R^{-1}$ on $\Delta M_{s}$. For this purpose, we would like to mention that $\left|V_{t s}\right|$ is very close to $\left|V_{c b}\right|$ due to CKM unitarity. As $\left|V_{c b}\right|$ is common for both the SM and nmUED scenario, therefore, $\left|V_{t s}\right|$ is common with an excellent accuracy to both models

\footnotetext{
${ }^{6}$ In view of the discussions given in Ref. [9] the effect of the KK-modes on the charm- and mixed charm-top contributions is totally insignificant. Considerable effect for these modes are only top contributions which has been indicated by the same function $S\left(x_{t}, r_{f}, r_{V}, R^{-1}\right)$ as in the case of $\Delta M_{q}$. Therefore, the $K_{L}-K_{S}$ mass difference $\Delta M_{K}$, is practically dominated by internal charm contributions and essentially unaffected by the KK-modes. Consequently, in the present nmUED scenario we have also found the same phenomenon. We hence refrain from providing the details of $\varepsilon_{K}$ in the present article.

${ }^{7}$ Here, we use $\sqrt{\hat{B}_{B_{d}}} F_{B_{d}}=(225 \pm 9) \mathrm{MeV}$ [62] and $m_{B_{d}}=$ $(5279.63 \pm 0.20) \mathrm{MeV}[62]$.
} 
and consequently we can have the following relation ${ }^{8}$

$$
\frac{\left(\Delta M_{S}\right)_{\mathrm{nmUED}}}{\left(\Delta M_{S}\right)_{\mathrm{SM}}}=\frac{S\left(x_{t}, r_{f}, r_{V}, R^{-1}\right)}{S_{0}\left(x_{t}\right)} .
$$

Let us focus on the above Eq. 37. It is clear from the Eq. 23 that the function $S\left(x_{t}, r_{f}, r_{V}, R^{-1}\right)$ (numerator of the right hand side of the Eq. 37) represents the combine contributions of SM and the NP (in the present case KK-contributions) obtained from the the box diagram given in Fig. 1. Besides, the function $S_{0}\left(x_{t}\right)$ (denominator of the right hand side of the Eq. 37) represents the SM contributions only. Moreover, from the above Eq. 37 it is obvious that the ratio of the function $S\left(x_{t}, r_{f}, r_{V}, R^{-1}\right)$ to the function $S_{0}\left(x_{t}\right)$ is equivalent to $\frac{\left(\Delta M_{s}\right)_{\text {muED }}}{\left(\Delta M_{s}\right)_{\text {SM }}}$. Therefore, from this ratio we have the opportunity to estimate the effects of BLT parameters $\left(r_{V}, r_{f}\right)$ and $R^{-1}$ by the virtue of $\Delta B=2$ transitions. We can compare this ratio 9 with the quantity $\frac{\left(\Delta M_{s}\right)_{\exp }}{\left(\Delta M_{s}\right)_{S M}}$. Now the value of SM prediction for $\Delta M_{S}$ is $(18.77 \pm 0.86) \mathrm{ps}^{-1}$ i.e., $\left(\Delta M_{S}\right)_{\mathrm{SM}}=$ $(18.77 \pm 0.86) \mathrm{ps}^{-1}$ [71]. On the other hand experimental value for the same is $(17.749 \pm 0.019 \pm 0.007) \mathrm{ps}^{-1}$, i.e., $\left(\Delta M_{s}\right)_{\exp }=(17.749 \pm 0.019 \pm 0.007) \mathrm{ps}^{-1}$ [62]. Note that in both cases the errors are given with $1 \sigma$ uncertainty. Now using these values we can easily determine the value ${ }^{10}$ of the ratio $\frac{\left(\Delta M_{s}\right)_{\mathrm{exp}}}{\left(\Delta M_{\mathrm{s}}\right)_{\mathrm{SM}}}$ and the corresponding value is $(0.946 \pm 0.043)$, i.e., $\frac{\left(\Delta M_{s}\right)_{\text {exp }}}{\left(\Delta M_{s}\right)_{S M}}=(0.946 \pm 0.043)$. In this case also the error is given with $1 \sigma$ uncertainty. However, while we perform the numerical analysis, we compare the quantity $\frac{\left(\Delta M_{s}\right)_{\text {muED }}}{\left(\Delta M_{s}\right)_{S M}}$ with the $2 \sigma$ allowed range of the obtained value of this ratio, i.e., $(0.946 \pm 0.086)$. Variation of this quantity with respect to $R^{-1}$ is given in Fig. 4e. With this treatment, we have estimated the effects of $R^{-1}$ and BLT parameters $\left(r_{V}, r_{f}\right)$ on the $\Delta M_{S}$ in nmUED scenario. We can also obtain lower limit on $R^{-1}$ form this ratio $\frac{\left(\Delta M_{s}\right)_{\mathrm{nmUED}}}{\left(\Delta M_{\mathrm{s}}\right)_{\mathrm{SM}}}$.

\section{Numerical analysis}

In the current article, for the first time we have computed the KK-contributions to the WC of the operator $(\Delta B=2)$ in the nmUED scenario. The function $S_{n}\left(x_{t}, x_{f^{(n)}}, x_{V^{(n)}}\right)$ given in Eq. 27 corresponds to the $n$th level KK-contributions to

\footnotetext{
${ }^{8}$ In view of the discussions given just before the Sect. 3.1, we assume that all other quantities like QCD factor, renormalisation group invariant parameter are same for both SM and nmUED scenarios.

9 Here, we would like to mention that, in order to measure the effect of Georgi-Machacek model on $\Delta M_{s}$, the same procedure has been adopted in Ref. [72].

${ }^{10}$ Since in this case $\left(\Delta M_{S}\right)_{\mathrm{SM}}$ and $\left(\Delta M_{S}\right)_{\exp }$ are independent quantities, therefore the uncertainty of the ratio $\frac{\left(\Delta M_{s}\right)_{\exp }}{\left(\Delta M_{s}\right)_{S M}}$ has been determined from the quadrature sum method. For the purpose of determination of the value of this quantity with uncertainty, we have followed the method given in the book, An introduction to Error Analysis, by John R. Taylor.
}

the coefficient for the $\Delta B=2$ operator. The function $S_{n}$ contains the dependence of KK-masses of gauge boson as well as fermion in the nmUED scenario. Besides, in view of the analysis of the effect of the SM Higgs mass on vacuum stability in UED model [73], we consider the sum of KKcontributions up to $5 \mathrm{KK}$-levels ${ }^{11}$ and thereafter we add up the total KK-contributions with the SM counterpart. ${ }^{12}$ Moreover, due to the converging ${ }^{13}$ nature of KK-summation, the numerical values would not differ drastically whether one considers higher numbers of KK-levels during the evaluation of KK-contributions for the loop diagrams [53-55].

\subsection{Possible constraints and range of BLT parameters}

Here we highlight the following constraints that have been considered in our analysis.

- In this nmUED scenario, comprehensive analyses on different rare $B$-decay processes (FCNC type), for example $B_{s} \rightarrow \mu^{+} \mu^{-}$[53], $B \rightarrow X_{s} \gamma$ [54] and $B \rightarrow X_{s} \ell^{+} \ell^{-}$ [55] have been performed. Moreover, these processes have always been played significant role for searching any favourable kind of NP scenario. In all these cases the expressions of these observables are the functions of the same set of parameters i.e., $r_{V}, r_{f}$ and $R^{-1}$ which are also involved in the expressions of the observables of the current article. Therefore, in this article it is very necessary that we should deal with such parameter space which is satisfied by the experimental data of these observables. Using the expressions of $\operatorname{Br}\left(B_{s} \rightarrow \mu^{+} \mu^{-}\right)$, $\operatorname{Br}\left(B \rightarrow X_{s} \gamma\right)$ and $\operatorname{Br}\left(B \rightarrow X_{s} \ell^{+} \ell^{-}\right)$given in [53-55] we have considered the branching ratios of these rare decay processes as constraints in our present study. In the following we present the latest experimental data for branching ratios of these processes

- Electroweak precision test (EWPT) is significant and instrumental for constraining any kind of BSM physics. Using the technique of the correction to Fermi constant $G_{F}$ at tree level one can perform the corrections to Peskin-Takeuchi parameters $\mathrm{S}, \mathrm{T}$, and $\mathrm{U}$ in the nmUED model. This is a distinctive feature of this nmUED scenario with respect to the minimal version of the UED model where these corrections emerge at one-loop processes. A detailed exercise on EWPT in this nmUED model has been given in $[53,56]$. The $\mathrm{S}, \mathrm{T}$, and $\mathrm{U}$ param-

\footnotetext{
11 In earlier studies, usually 20-30 KK-levels have been taken while adding up the contributions from KK-modes.

12 We have taken $M_{W}=80.379 \mathrm{GeV}$ [62] for SM $W^{ \pm}$boson mass and $\bar{m}_{t}\left(m_{t}\right)=165.25 \mathrm{GeV}$ [63] for SM top quark mass.

13 The summation of KK-contribution is convergent in UED type models with one extra space-like dimension, as far as one-loop calculation is concerned [74].
} 
eters in the nmUED model are the functions of $r_{V}, r_{f}$ and $R^{-1}$. Following the similar approach provided in refs. $[53,56]$ we have imposed EWPT as one of the constraints in the analysis.

To this end, we would like to discuss the range of values of BLT parameters used in our present exercise. In general values of the BLT parameters may be negative or positive. However, it is clearly seen from Eq. 7 that, for $r_{f} / R=-\pi$ the zero-mode solution becomes divergent and beyond $r_{f} / R=-\pi$ the zero-mode fields become ghost-like. Therefore, any values of BLT parameters lower than $-\pi R$ should not be considered, but for the purpose of completeness we have taken some negative values of BLT parameters in our numerical analysis. However, from the study of electroweak precision data $[53,56]$ large portion of negative values of BLT parameters have been disfavoured.

\subsection{Results}

At this stage, considering the above mentioned constraints, we would like to find the parameter space which satisfy the $2 \sigma$ allowed ranges of the CKM elements and $\frac{\left(\Delta M_{s}\right)_{\text {exp }}}{\left(\Delta M_{s}\right)_{S M}}$ simultaneously. In the present version of nmUED scenario we have three independent free parameters e.g., inverse of radius of compactification $R^{-1}$, dimensionless scaled BLT parameters for boson $R_{V}\left(=r_{V} / R\right)$ and fermion $R_{f}\left(=r_{f} / R\right)$. In order to find the allowed parameter space we have chosen the following ranges for the free parameters:

$R^{-1} \in[0.05,2] \mathrm{TeV} ; R_{V} \in[-3,20]$ and $R_{f} \in[-3,20]$.

Using the above ranges of free parameters we have obtained a region in $R_{V}-R_{f}$ plane shown in Fig. 3. Moreover, for different combination of $R_{V}, R_{f}$ the allowed values of $R^{-1}$ are displayed by colour codes. The Fig. 3 shows that, with the increasing values of $R_{V}$ and $R_{f}$ the allowed values of $R^{-1}$ are increased. This can be explained in the following way. Since with the increasing values of BLT parameters the KK-masses are decreased, consequently with the decreasing values of KK-masses the loop function $S$ (obtained from oneloop box diagrams) is enhanced. Therefore, in order to compensate this enhancement one requires the increasing values of $R^{-1}$ (as KK-masses are increased with the increasing values of $R^{-1}$ ). Another notable feature is that for $R_{V} \approx R_{f}$ most of the allowed values of $R^{-1}$ with higher in magnitude are appeared and these type of points are increased with the larger values of BLT parameters. Moreover, it is evident from the figure that only a few portion of negative values of BLT parameters are allowed. The reason is that, apart from the constraints on branching ratio of several rare $B$-decay process we have considered EWPT as crucial one. As a consequences, in this nmUED scenario EWPT favours the region

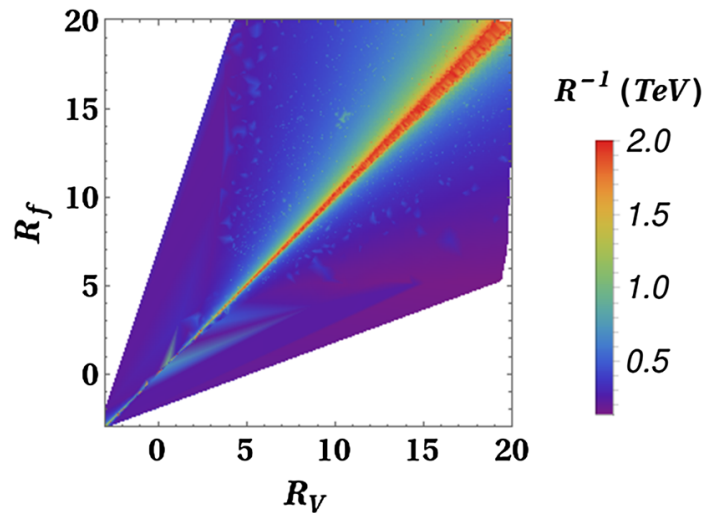

Fig. 3 Allowed parameter space in $R_{V}\left(=r_{V} / R\right)-R_{f}\left(=r_{f} / R\right)$ plane satisfying $2 \sigma$ range of latest values of CKM elements and $\frac{\left(\Delta M_{s}\right)}{\left(\Delta M_{s}\right)_{\mathrm{SM}}}$ simultaneously. Moreover, the allowed values of $R^{-1}$ for different combination of $R_{V}\left(=r_{V} / R\right), R_{f}\left(=r_{f} / R\right)$ are indicated by colour codes. Here we sum the contributions up to 5 KK-levels in loop function $S$ while calculating box diagram

where $R_{V} \approx R_{f}$ and larger values of $R^{-1}$ are disfavoured for most of the negative values of BLT parameters $[53,56]$. Furthermore, we would like to mention that, among the different constraints of $B$-physics observables $\left(\operatorname{Br}\left(B_{s} \rightarrow \mu^{+} \mu^{-}\right)\right.$ [53], $\operatorname{Br}\left(B \rightarrow X_{s} \gamma\right)$ [54] and $\operatorname{Br}\left(B \rightarrow X_{s} \ell^{+} \ell^{-}\right)$[55] $)$ $\operatorname{Br}\left(B_{s} \rightarrow \mu^{+} \mu^{-}\right)[53]$ is the most dominating in nature. Therefore, as far as the $B$-physics is concerned, before the present analysis of $\Delta B=2$ transitions of this article, the most admissible value of lower limit of $R^{-1}$ was derived from the $\operatorname{Br}\left(B_{s} \rightarrow \mu^{+} \mu^{-}\right)$[53] for a specific combination of BLT parameter in the present version of nmUED scenario. However, from the current analysis of $\Delta B=2$ transitions, we have found a distinguishable observation with respect to our previous analyses on rare $B$-decay process [53-55]. For example, in contrast to the previous analyses [53-55], here we obtain lower limit of $R^{-1}$ with larger values for several combination of positive values of scaled BLT parameters $\left(R_{V}, R_{f}\right)$. In the following, for the purpose of illustration, we have picked up two sets of nonvanishing scaled BLT parameters $\left(R_{V}, R_{f}\right)$ from the allowed region shown in the Fig. 3 and consequently using the Fig. 4 we will discuss the characteristic dependence of the observables which have been considered in this article with respect to $R^{-1}$. Moreover, depending on the BLT parameters we can estimate the lower bound on the $R^{-1}$ (using the Fig. 4) that are obtained from the present analysis of $\Delta B=2$ transition.

The Fig. 4 contains 5 panels that have shown the dependence of the variables $\left|V_{t d}\right|, \gamma$ (in degree), $\bar{\eta}, \bar{\rho}$ and $\frac{\left(\Delta M_{s}\right)}{\left(\Delta M_{s}\right) \text { SM }}$ with respect to $R^{-1}$. In each panel we have shown the dependence of these observables for three benchmark points which have been chosen from the allowed parameter space shown in Fig. 3. The first benchmark point (BP1) is indicated by the red coloured solid line and for this case $R_{V}=6.72$ and 


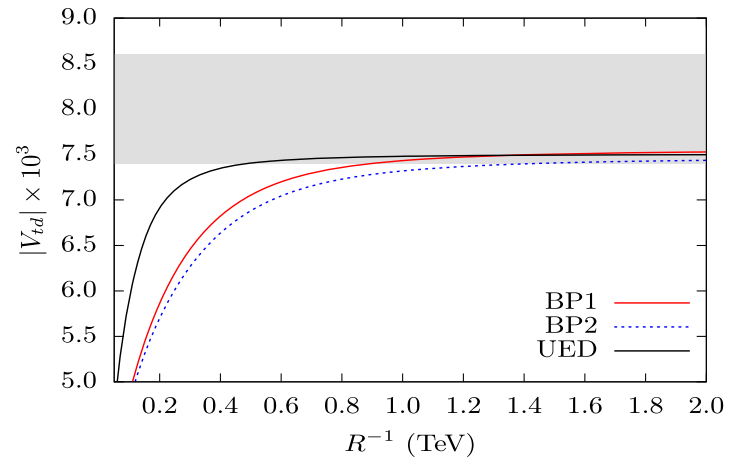

(a)

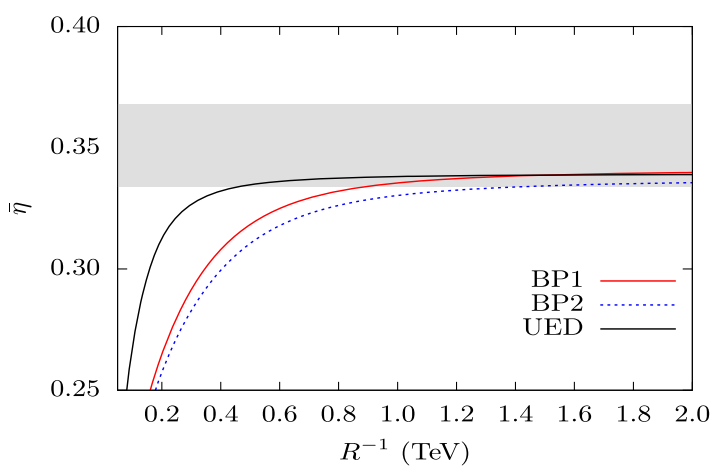

(c)

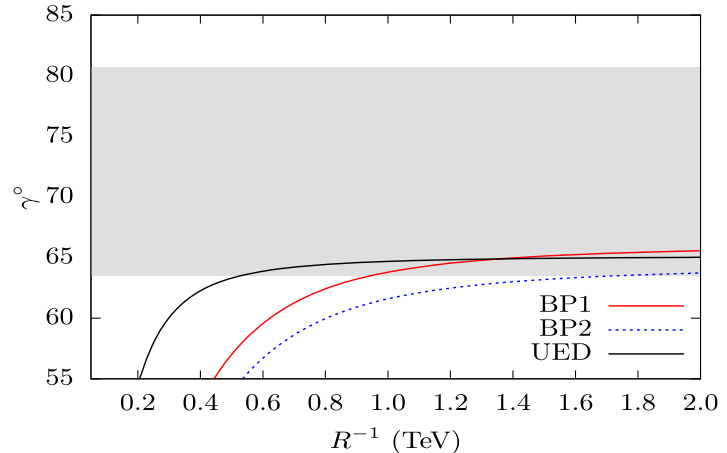

(b)

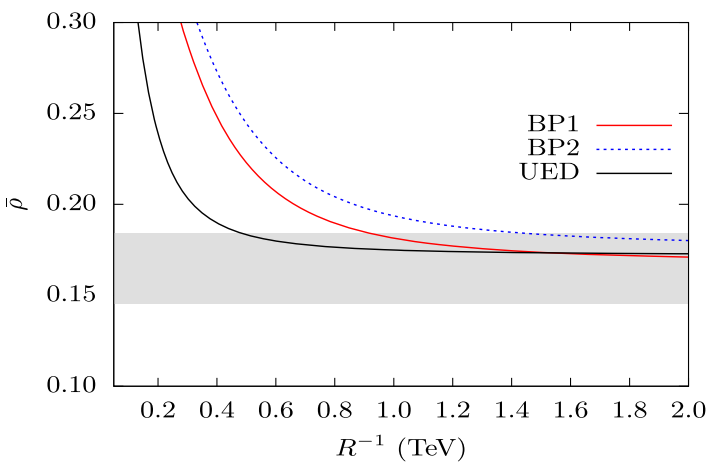

(d)

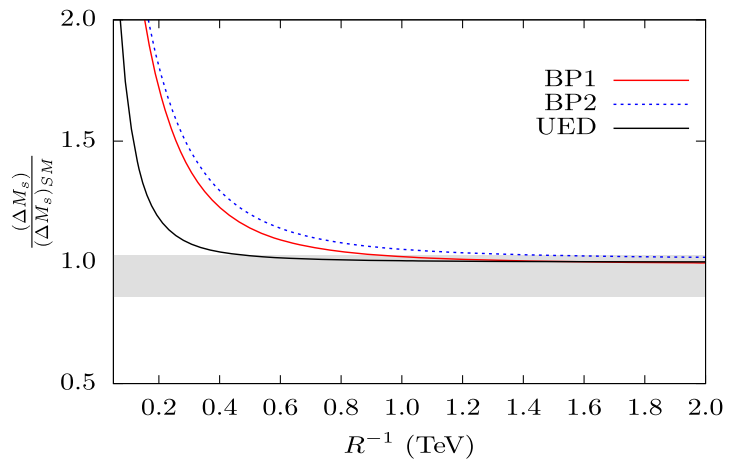

(e)

Fig. 4 Variation of CKM parameters $\left[\mathbf{a}\left|V_{t d}\right| ; \mathbf{b} \gamma^{\circ} ; \mathbf{c} \bar{\eta} ; \mathbf{d} \bar{\rho}\right]$ and $\mathbf{e}$ $\frac{\left(\Delta M_{s}\right)}{\left(\Delta M_{s}\right)_{S M}}$ with respect to $R^{-1}$, where we have chosen three sets of scaled BLT parameters $R_{V}\left(=r_{V} / R\right)=6.72, R_{f}\left(=r_{f} / R\right)=6.58$ (considered as BP1 indicated by solid red line); $R_{V}\left(=r_{V} / R\right)=8.76, R_{f}(=$ $\left.r_{f} / R\right)=9.06$ (considered as BP2 indicated by doted blue line) and
$R_{V}\left(=r_{V} / R\right)=0, R_{f}\left(=r_{f} / R\right)=0$ (considered as UED indicated by solid black line) from the allowed parameter space as shown in the Fig. 3. Here we sum the contributions up to $5 \mathrm{KK}$-levels in loop function $S$ while calculating box diagrams. The horizontal gray band shows the $2 \sigma$ allowed range of respective observables
Table 1 Experimental data for branching ratios of

$$
\begin{aligned}
& B_{s} \rightarrow \mu^{+} \mu^{-}, B \rightarrow X_{s} \gamma \text { and } \\
& B \rightarrow X_{s} \ell^{+} \ell^{-}
\end{aligned}
$$

\begin{tabular}{|c|c|c|}
\hline Observables & & Experimental value \\
\hline $\operatorname{Br}\left(B_{s} \rightarrow \mu^{+} \mu^{-}\right)$ & & $(3.1 \pm 0.6) \times 10^{-9}[70]$ \\
\hline $\operatorname{Br}\left(B \rightarrow X_{s} \gamma\right)$ & & $(3.32 \pm 0.15) \times 10^{-4}[70]$ \\
\hline $\operatorname{Br}\left(B \rightarrow X_{s} \ell^{+} \ell^{-}\right)$ & $\begin{array}{l}q^{2} \in[1,6] \mathrm{GeV}^{2} \\
q^{2} \in[14.4,25] \mathrm{GeV}^{2}\end{array}$ & $\begin{array}{l}\left(1.60_{-0.39-0.13}^{+0.41+0.17} \pm 0.18\right) \times 10^{-6}[75] \\
\left(0.57_{-0.15-0.02}^{+0.16+0.03} \pm 0.00\right) \times 10^{-6}[75]\end{array}$ \\
\hline
\end{tabular}


Table 2 Lower limits on $R^{-1}$ (in $\mathrm{GeV}$ ) evaluated for various CKM parameters $\left[\left|V_{t d}\right|, \gamma\right.$ (in degree), $\left.\bar{\eta}, \bar{\rho}\right]$ and $\frac{\left(\Delta M_{s}\right)}{\left(\Delta M_{s}\right)_{S M}}$ for the selected benchmark points (BP1 and BP2) and UED. For each panel in Fig. 4, the lower limits (on $R^{-1}$ ) have been obtained from intersection of the respective curves with the $2 \sigma$ allowed range of the corresponding observables

\begin{tabular}{lrrrrr}
\hline & From $\left|V_{t d}\right|$ & From $\gamma^{\circ}$ & From $\bar{\eta}$ & From $\bar{\rho}$ & From $\frac{\left(\Delta M_{s}\right)}{\left(\Delta M_{s}\right) \text { SM }}$ \\
\hline BP1 & 898.56 & 952.09 & 888.32 & 922.74 & 895.09 \\
BP2 & 1381.58 & 1482.38 & 1329.05 & 1418.51 & 1335.04 \\
UED & 482.20 & 535.48 & 461.41 & 488.96 & 469.95 \\
\hline
\end{tabular}

$R_{f}=6.58$. The second benchmark point (BP2) is indicated by the blue coloured dashed line and for this case $R_{V}=8.76$ and $R_{f}=9.06$. Finally, in order to show the UED results we have taken another point (also resides within the region shown in Fig. 3) which is indicated by black coloured solid line for which $R_{V}=0$ and $R_{f}=0$.

Let us focus on the BP1. For this benchmark point, when the curve of each observables intersects the $2 \sigma$ allowed range of the corresponding observables, then we obtain the values of lower limits of $R^{-1}$ for the observables. These values of lower limits are given in Table $2 \mathrm{in} \mathrm{GeV}$ unit. From this table it is clear that, for BP1 the highest value of lower limit of $R^{-1}$ is obtained from the observable $\gamma$ (e.g., $952.09 \mathrm{GeV}$ ) while the lowest value of lower limit is generated from the observable $\bar{\eta}$ (e.g., $888.32 \mathrm{GeV}$ ). Let us now discuss the nature of these curves. First of all, using the Eq. 28 we have derived the value of $\left|V_{t d}\right|$ and it is governed by the function $S$ which is dependent on the model parameters $R_{V}, R_{f}$ and $R^{-1}$. Now for a fixed value of $R_{V}$ and $R_{f}$ the KK-masses are controlled by $R^{-1}$. With the increasing values of $R^{-1}$ the KK-masses are increased, hence the loop function $S$ is decreased. Therefore, naturally, the value of $\left|V_{t d}\right|$ is increased with $R^{-1}$. However, after a certain value of $R^{-1}$, when the KK-masses are very high then decoupling behavior of the KK-mode contribution arises and consequently there is no variation of $\left|V_{t d}\right|$ with $R^{-1}$. After determination of $\left|V_{t d}\right|$ we have derived $R_{t}$ using Eq. 33. Thereafter, with this $R_{t}$ we have derived $\bar{\rho}, \bar{\eta}$ and $\gamma$ from the Eq. 36. It is evident from Eq. 33, that for the given values of $\lambda$ and $V_{c b}, R_{t}$ is proportional to $\left|V_{t d}\right|$, therefore $R_{t}$ will follow the same characteristics as $\left|V_{t d}\right|$ with $R^{-1}$. Moreover, it is also evident from the Eq. 36 that $\bar{\eta}$ is proportional to $R_{t}$ (for a given value of $\beta$ ), therefore, $\bar{\eta}$ will be increased with the increasing values of $R^{-1}$ and obviously $\bar{\rho}$ will be decreased. Further, the increasing behaviour of $\gamma$ with respect to $R^{-1}$ is a resultant factor of increment of $\bar{\eta}$ (with $R^{-1}$ ) and decrement of $\bar{\rho}$ (with $R^{-1}$ ). At the KK-mode decoupling limit, the values of all observables are saturated and do not show any variation with $R^{-1}$. Now the behaviour of the curve given in the last panel (4e) of the Fig. 4 can be explained in the following way. As we have already mentioned that the nmUED scenario is a class of MFV models, therefore, the NP contribution to one-loop box diagram is always positive. Hence, $\frac{\left(\Delta M_{s}\right)}{\left(\Delta M_{s}\right)_{\mathrm{SM}}}$ (for the sake of notational simplicity here we assume, $\left(\Delta M_{S}\right) \equiv\left(\Delta M_{S}\right)_{\text {nmUED }}$, while $\left.\left(\Delta M_{S}\right)=\left(\Delta M_{S}\right)_{\mathrm{SM}}+\left(\Delta M_{S}\right)_{\mathrm{NP}}\right)$ is greater than one. Further, with the increasing values of $R^{-1}$ (basically KK-mass) the one-loop function $S$ is decreased, therefore, the quantity $\frac{\left(\Delta M_{S}\right)}{\left(\Delta M_{S}\right)_{S M}}$ is decreased and after a certain large value of $R^{-1}$ it will become one.

All the above mentioned explanations for all the observables also hold good for BP2. However, for BP2 the lower limits on $R^{-1}$ are changed and they are also presented in Table 2 in GeV unit. From the values of lower limits of $R^{-1}$ (that are given in Table 2 for BP2) it is clear that the lower limits of $R^{-1}$ for BP2 are larger. As for example the highest value (which is obtained from $\gamma$ ) is $1482.38 \mathrm{GeV}$ and the lowest value (which is obtained from $\bar{\eta}$ ) is $1329.05 \mathrm{GeV}$. This enhancement of lower limit with respect to the BP1 can easily be explained in the following way. Since in the case of $\mathrm{BP} 2$, the values of the BLT parameter are higher than that of the BP1, therefore the KK-masses are decreased. Hence, in order to compensate the KK-mass decrement larger value of $R^{-1}$ is required. Therefore, for BP2 we obtain larger values of lower limits on $R^{-1}$ for all observables.

At this stage we would like to emphasize that the current analysis of $\triangle B=2$ transitions provide us better result with respect to our earlier analyses [53-55] on rare decays of $B$ meson in the same version of nmUED scenario. From the previous discussions it is already clear that we can push the lower limit on $R^{-1}$ to appreciable higher range $(\approx 1.48 \mathrm{TeV}$ or even higher) for favourable choice BLT parameters. On the other hand, for illustrative purpose we have explicitly checked the lower limits of $R^{-1}$ for two benchmark points (BP1 and BP2) for $\operatorname{Br}\left(B_{S} \rightarrow \mu^{+} \mu^{-}\right)$. The values of the lower limits are lesser than that obtained from the current analysis of $\Delta B=2$ transitions. For example for BP1 the lower limit of $R^{-1}$ from $\operatorname{Br}\left(B_{s} \rightarrow \mu^{+} \mu^{-}\right)$is $841 \mathrm{GeV}$ while for BP2 the corresponding value is $1.26 \mathrm{TeV} .{ }^{14}$ Hence, comparing these two values of lower limit of $R^{-1}$ with the same obtained from the analysis of $\Delta B=2$ transition (e.g., $952.09 \mathrm{GeV}$ for $\mathrm{BP} 1$ and $1.48 \mathrm{TeV}$ for $\mathrm{BP} 2$ ) we can readily infer that we

\footnotetext{
${ }^{14}$ Here, among the all $B$-physics constraints that have been considered in the present article, we have mentioned the lower limit of $R^{-1}$ for two benchmark points for $\operatorname{Br}\left(B_{s} \rightarrow \mu^{+} \mu^{-}\right)$only. Before the present analysis of $\Delta B=2$ transitions, the most dominant constraint came from $\operatorname{Br}\left(B_{s} \rightarrow \mu^{+} \mu^{-}\right)$in nmUED scenario.
} 
have obtained so far the most dominating constraining power from our present analysis of $\Delta B=2$ transitions. Therefore, from $B$-physics perspective, these lower limits, that we have obtained from the present analysis of $\Delta B=2$ transitions, are so far the most admissible results in the current version of nmUED scenario.

Before we conclude, we would like to remark on the lower limits on $R^{-1}$ which are achieved in the UED scenario considering the current analysis on $\Delta B=2$ transitions. We can achieve the UED results from our analysis when BLT parameters vanish, i.e., for $R_{V}=R_{f}=0$. In this set up KK-mass for $n$th KK-level simply emerges as $n R^{-1}$. On the other hand, the overlap integrals $I_{1}^{n}$ and $I_{2}^{n}$ become unity. Hence, with this limit, the function $S_{n}\left(x_{t}, x_{f^{(n)}}, x_{V^{(n)}}\right)$ is converted into its UED form. We have independently checked that when the BLT parameters are zero the expressions of the function $S_{n}\left(x_{t}, x_{f^{(n)}}, x_{V^{(n)}}\right)$ is exactly matched with that of the given in Ref. [9]. ${ }^{15}$ Like the chosen benchmark points BP1 and BP2 the values of lower limits of $R^{-1}$ for $R_{V}=R_{f}=0$ are also presented in Table 2 . In this case the highest value (which is obtained from $\gamma$ ) is $535.48 \mathrm{GeV}$ and the lowest value (which is obtained from $\bar{\eta}$ ) is $461.41 \mathrm{GeV}$. Considering $B$-physics analyses, these values of lower limits of $R^{-1}$ are slightly improved, because the value of these lower limits are slightly higher than that obtained from our previous analyses [53-55]. However, it is needless to say that, these limits are not very striking values, but close to those values that have been achieved from previous analyses in UED scenario. As for example $(g-2)_{\mu}$ [76], $\rho$-parameter [77], FCNC process $[8,9,78,79], Z b \bar{b}[52,80]$ and electroweak observables [8183] provide a lower bound of about $300-600 \mathrm{GeV}$ on $R^{-1}$. Besides, from the analysis of projected tri-lepton signal at 8 TeV LHC one can obtain lower limit on $R^{-1}$ up to $1.2 \mathrm{TeV}$ [84-86]. At this stage it is needed to mention that the value of lower limits on $R^{-1}$ from the analysis on $\Delta B=2$ transitions for minimal version of UED scenario, have already been excluded by the LHC data. Since the recent analyses including LHC data have ruled out $R^{-1}$ up to $1.4 \mathrm{TeV}$ [8790].

\section{Summary}

In this article we estimate the Kaluza-Klein contribution to the $\Delta B=2$ transitions in a class of (4+1)-dimensional Universal Extra Dimensional (in which all Standard Model particle can propagate along the extra spatial dimension) scenario in the presence of boundary localised terms (BLTs). The coefficient of these terms are parametrised to the unknown

\footnotetext{
${ }^{15}$ In the article [9], the authors have not considered any radiative corrections to the KK-masses in their analysis. Therefore, the KK-mass at the $n$th KK-mode is $n R^{-1}$.
}

radiative corrections for the masses and couplings of KaluzaKlein modes. Due to the presence of these boundary terms the masses and coupling strengths are nontrivially modified in 4-dimensional effective theory with respect to the minimal version of the Universal Extra Dimensional scenario. Utilising two different kinds of BLT parameters e.g., $r_{V}$ (represents the coefficients of boundary terms for the gauge and Higgs sectors) and $r_{f}$ (specifies the coefficients of boundary terms of fermions and Yukawa interactions) we have investigated the $\Delta B=2$ transitions in nonminimal Universal Extra Dimensional scenario.

The effective Hamiltonian for the $\Delta B=2$ transitions can be expressed by four-fermion interactions and the coefficient of the interactions are parametrised by appropriate Wilson Coefficient. With the computation of one-loop box diagrams given in Fig. 1 we have evaluated the coefficient for the operator that is responsible for the $\Delta B=2$ transitions. Moreover, utilising the Glashow Iliopoulos Maiani mechanism we have included contributions from three generations of quarks in our analysis. On the other hand, considering a recent analysis relating the Higgs boson mass and cut-off of a universal extra dimensional theory [73] we summed up to five Kaluza-Klein modes in our computation. Further, in view of the fact that the nonminimal universal extra dimensional scenario belongs to a class of minimal flavour violation models, we have simply added the Kaluza-Klein contributions coming from the oneloop box diagrams (Fig. 1) to the corresponding Standard Model (zero-mode) contributions.

After evaluation of the function $S$ (obtained from oneloop box diagrams) we have determined several elements e.g., $\left|V_{t d}\right|, \bar{\eta}, \bar{\rho}$ and $\gamma$ in nonminimal Universal Extra Dimensional scenario. Some of these quantities have played very important role for Wolfenstein parametrisation and further using these quantities we can estimate geometrical shape of unitarity triangle. Finally, we have evaluated the quantity $\Delta M_{S}$ scaled by the corresponding Standard Model value. Comparing our theoretical predictions of these quantities with the corresponding $2 \sigma$ allowed ranges, we have constrained the parameter space of the present version of nonminimal Universal Extra Dimensional scenario. Moreover, in our analysis we have considered the branching ratios of some important rare decay processes of $B$-meson: such as $B_{s} \rightarrow \mu^{+} \mu^{-}, B \rightarrow X_{s} \gamma$ and $B \rightarrow X_{s} \ell^{+} \ell^{-}$as well as electroweak precision data as constraints.

It has already been alluded that for the vanishing BLT parameters (i.e., $R_{f}=R_{V}=0$ ) we can reproduce the results of the minimal version of Universal Extra Dimensional scenario. Therefore, using our analysis, we have reexamined the lower limit on $R^{-1}$ in the framework of minimal Universal Extra Dimensional scenario in the vanishing BLT limit. In that case the value of the lower limit on $R^{-1}$ becomes 535.48 $\mathrm{GeV}$ (highest value that we have obtained from our present analysis of $\triangle B=2$ transition for UED) and it is slightly 
higher than those values that are determined from our earlier studies on $B$-physics. However, this value is excluded from recent collider analysis at the LHC.

Nevertheless, in the presence of different nonvanishing BLT parameters we can enrich the results of lower limit on $R^{-1}$ in the current version of nonminimal Universal Extra Dimensional scenario. As for example if we choose a set of BLT parameter $\left(R_{V}=6.72\right.$ and $R_{f}=6.58$, i.e., for BP1) from the allowed parameter space given in Fig. 3 then using our analysis we can obtain the lower limit of $R^{-1} \approx 952$ $\mathrm{GeV}$ (highest value that we have obtained from our present analysis of $\Delta B=2$ transition for BP1). Moreover, the same can be even higher (e.g., $\approx 1.48 \mathrm{TeV}$ ) if we choose another set of BLT parameter $\left(R_{V}=8.76\right.$ and $R_{f}=9.06$, i.e., for BP2) from the allowed parameter space shown in Fig. 3. Definitely, these results (lower limits on $R^{-1}$ ) in the current version of nonminimal universal extra dimensional scenario are the most admissible values in comparison to the limits obtained from our earlier analyses on rare decays of $B$-meson [53-55].

Acknowledgements The author would like to give thanks to Anirban Biswas for computational support. The author thanks Anindya Datta for some illuminating suggestions.

Data Availability Statement This manuscript has no associated data or the data will not be deposited. [Authors' comment: Plots and tables are self-sufficient and self-explanatory, therefore, no data is required to be deposited.]

Open Access This article is licensed under a Creative Commons Attribution 4.0 International License, which permits use, sharing, adaptation, distribution and reproduction in any medium or format, as long as you give appropriate credit to the original author(s) and the source, provide a link to the Creative Commons licence, and indicate if changes were made. The images or other third party material in this article are included in the article's Creative Commons licence, unless indicated otherwise in a credit line to the material. If material is not included in the article's Creative Commons licence and your intended use is not permitted by statutory regulation or exceeds the permitted use, you will need to obtain permission directly from the copyright holder. To view a copy of this licence, visit http://creativecomm ons.org/licenses/by/4.0/.

Funded by SCOAP ${ }^{3}$.

\section{Appendices}

\section{A Feynman rules required for the study of $\Delta B=2$ transitions in nmUED}

This appendix contains the Feynman rules required for our calculations with the assumption that all momenta and fields are incoming:

1) $G^{\mu} \bar{f}_{1} f_{2}: i g_{s} T_{\alpha \beta}^{a} \gamma_{\mu} C$, where $C$ takes the following form:

$$
G^{\mu} \bar{u}_{i} u_{i}: C=1
$$

$G^{\mu} \bar{T}_{i}^{1(n)} T_{i}^{1(n)}: C=1$,

$G^{\mu} \bar{T}_{i}^{2(n)} T_{i}^{2(n)}: C=1$,

$G^{\mu} \bar{T}_{i}^{1(n)} T_{i}^{2(n)}: C=0$,

$G^{\mu} \bar{T}_{i}^{2(n)} T_{i}^{2(n)}: C=0$.

2) $S^{ \pm} \bar{f}_{1} f_{2}=\frac{g_{2}}{\sqrt{2} M_{W^{(n)}}}\left(P_{L} C_{L}+P_{R} C_{R}\right)$, where $C_{L}$ and $C_{R}$ are expressed in the following way:

$$
\begin{aligned}
& G^{+} \overline{u_{i}} d_{j}:\left\{\begin{array}{l}
C_{L}=-m_{i} V_{i j}, \\
C_{R}=m_{j} V_{i j},
\end{array} G^{-} \bar{d}_{j} u_{i}:\left\{\begin{array}{l}
C_{L}=-m_{j} V_{i j}^{*}, \\
C_{R}=m_{i} V_{i j}^{*},
\end{array}\right.\right. \\
& G^{(n)+} \bar{T}_{i}^{1(n)} d_{j}:\left\{\begin{array}{l}
C_{L}=-m_{1}^{(i)} V_{i j}, \\
C_{R}=M_{1}^{(i, j)} V_{i j},
\end{array} \quad G^{(n)-} \bar{d}_{j} T_{i}^{1(n)}:\left\{\begin{array}{l}
C_{L}=-M_{1}^{(i, j)} V_{i j}^{*}, \\
C_{R}=m_{1}^{(i)} V_{i j}^{*},
\end{array}\right.\right. \\
& G^{(n)+} \bar{T}_{i}^{2(n)} d_{j}:\left\{\begin{array}{l}
C_{L}=m_{2}^{(i)} V_{i j}, \\
C_{R}=-M_{2}^{(i, j)} V_{i j},
\end{array} \quad G^{(n)-} \bar{d}_{j} T_{i}^{2(n)}:\left\{\begin{array}{l}
C_{L}=M_{2}^{(i, j)} V_{i j}^{*}, \\
C_{R}=-m_{2}^{(i)} V_{i j}^{*},
\end{array}\right.\right. \\
& H^{(n)+} \bar{T}_{i}^{1(n)} d_{j}:\left\{\begin{array}{l}
C_{L}=-m_{3}^{(i)} V_{i j}, \\
C_{R}=M_{3}^{(i, j)} V_{i j},
\end{array} \quad H^{(n)-} \bar{d}_{j} T_{i}^{1(n)}:\left\{\begin{array}{l}
C_{L}=-M_{3}^{(i, j)} V_{i j}^{*}, \\
C_{R}=m_{3}^{(i)} V_{i j}^{*},
\end{array}\right.\right. \\
& H^{(n)+} \bar{T}_{i}^{2(n)} d_{j}:\left\{\begin{array}{l}
C_{L}=m_{4}^{(i)} V_{i j}, \\
C_{R}=-M_{4}^{(i, j)} V_{i j},
\end{array} \quad H^{(n)-} \bar{d}_{j} T_{i}^{2(n)}:\left\{\begin{array}{l}
C_{L}=M_{4}^{(i, j)} V_{i j}^{*}, \\
C_{R}=-m_{4}^{(i)} V_{i j}^{*} .
\end{array}\right.\right.
\end{aligned}
$$

3. $W^{\mu \pm} \bar{f}_{1} f_{2}: \frac{i g_{2}}{\sqrt{2}} \gamma_{\mu} P_{L} C_{L}$, where $C_{L}$ takes the following form [53]:

$$
\begin{aligned}
& W^{\mu+} \bar{u}_{i} d_{j}: \quad C_{L}=V_{i j}, \quad W^{\mu-} \bar{d}_{j} u_{i}: \quad C_{L}=V_{i j}^{*}, \\
& W^{\mu(n)+} \bar{T}_{i}^{1(n)} d_{j}: \quad C_{L}=I_{1}^{n} c_{i n} V_{i j}, \quad W^{\mu(n)-} \bar{d}_{j} T_{i}^{1(n)}: \\
& \quad C_{L}=I_{1}^{n} c_{i n} V_{i j}^{*}, \\
& W^{\mu(n)+} \bar{T}_{i}^{2(n)} d_{j}: C_{L}=-I_{1}^{n} s_{i n} V_{i j}, \quad W^{\mu(n)-} \bar{d}_{j} T_{i}^{2(n)}: \quad C_{L}=-I_{1}^{n} s_{i n} V_{i j}^{*},
\end{aligned}
$$

where the fermion fields $f \equiv u, d, T_{t}^{1}, T_{t}^{2}$.

The mass parameters $m_{x}^{(i)}$ are expressed in the following way [53]:

$m_{1}^{(i)}=I_{2}^{n} m_{V^{(n)}} c_{i n}+I_{1}^{n} m_{i} s_{i n}$,

$m_{2}^{(i)}=-I_{2}^{n} m_{V^{(n)}} s_{i n}+I_{1}^{n} m_{i} c_{i n}$,

$m_{3}^{(i)}=-I_{2}^{n} i M_{W} c_{i n}+I_{1}^{n} i \frac{m_{V^{(n)}} m_{i}}{M_{W}} s_{i n}$,

$m_{4}^{(i)}=I_{2}^{n} i M_{W} s_{i n}+I_{1}^{n} i \frac{m_{V^{(n)}} m_{i}}{M_{W}} c_{i n}$

where $m_{i}$ is identified as the mass of the zero-mode up-type fermion and $c_{i n}=\cos \left(\alpha_{i n}\right)$ and $s_{i n}=\sin \left(\alpha_{i n}\right)$ with $\alpha_{i n}$ as defined earlier.

And the mass parameters $M_{x}^{(i, j)}$ are expressed in the following way [53]:

$$
\begin{aligned}
& M_{1}^{(i, j)}=I_{1}^{n} m_{j} c_{i n}, \\
& M_{2}^{(i, j)}=I_{1}^{n} m_{j} s_{i n}, \\
& M_{3}^{(i, j)}=I_{1}^{n} i \frac{m_{V^{(n)}} m_{j}}{M_{W}} c_{i n},
\end{aligned}
$$


$M_{4}^{(i, j)}=I_{1}^{n} i \frac{m_{V^{(n)}} m_{j}}{M_{W}} s_{i n}$,

where $m_{j}$ is identified as the mass of the zero-mode downtype fermion.

In all the Feynman vertices the factors $I_{1}^{n}$ and $I_{2}^{n}$ are identified as the overlap integrals given in the following [53]

$$
\begin{aligned}
I_{1}^{n}= & \sqrt{\frac{1+\frac{r_{V}}{\pi R}}{1+\frac{r_{f}}{\pi R}}}\left[\frac{1}{\sqrt{1+\frac{r_{f}^{2} m_{f^{(n)}}^{2}}{4}+\frac{r_{f}}{\pi R}}}\right] \frac{m_{V^{(n)}}^{2}}{\left(m_{V^{(n)}}^{2}-m_{f^{(n)}}^{2}\right)} \frac{\left(r_{f}-r_{V}\right)}{\pi R}, \\
& \times\left[\frac{1}{\sqrt{1+\frac{r_{V}^{2} m_{V(n)}^{2}}{4}+\frac{r_{V}}{\pi R}}}\right] \\
I_{2}^{n}= & \sqrt{\frac{1+\frac{r_{V}}{1+\frac{r_{f}}{\pi R}}}{\pi R}}\left[\frac{1}{\sqrt{1+\frac{r_{f}^{2} m_{f^{(n)}}^{2}}{4}+\frac{r_{f}}{\pi R}}}\right] \\
& \times\left[\frac{1}{\left.\sqrt{1+\frac{r_{V}^{2} m_{V^{(n)}}^{2}+\frac{r_{V}}{\pi R}}{4}}\right] \frac{m_{V^{(n)}} m_{f^{(n)}}}{\left(m_{V^{(n)}}^{2}-m_{f^{(n)}}^{2}\right)} \frac{\left(r_{f}-r_{V}\right)}{\pi R} .} .\right.
\end{aligned}
$$

\section{References}

1. ATLAS collaboration, G. Aad et al., Observation of a new particle in the search for the Standard Model Higgs boson with the ATLAS detector at the LHC. Phys. Lett. B 716, 1-29 (2012). https://doi. org/10.1016/j.physletb.2012.08.020. arXiv:1207.7214

2. CMS collaboration, S. Chatrchyan et al., Observation of a New Boson at a Mass of $125 \mathrm{GeV}$ with the CMS Experiment at the LHC. Phys. Lett. B 716, 30-61 (2012). https://doi.org/10.1016/j. physletb.2012.08.021. arXiv:1207.7235

3. T. Inami, C.S. Lim, Effects of superheavy quarks and leptons in low-energy weak processes $K_{L} \rightarrow \mu \bar{\mu}, K^{+} \rightarrow \pi^{+} \nu \bar{\nu}$ and $K^{0} \leftrightarrow$ $\bar{K}^{0}$. Prog. Theor. Phys. 65(01), 297-314 (1981). https://doi.org/10. 1143/PTP.65.297

4. N. Cabibbo, Unitary symmetry and leptonic decays. Phys. Rev. Lett. 10, 531-533 (1963). https://doi.org/10.1103/PhysRevLett.10. 531

5. M. Kobayashi, T. Maskawa, CP-Violation in the renormalizable theory of weak interaction. Prog.Theor. Phys. 49(02), 652-657 (1973). https://doi.org/10.1143/PTP.49.652

6. A.J. Buras, P. Gambino, M. Gorbahn, S. Jager, L. Silvestrini, Universal unitarity triangle and physics beyond the standard model. Phys. Lett. B 500, 161-167 (2001). https://doi.org/10.1016/ S0370-2693(01)00061-2. arXiv:hep-ph/0007085

7. L. Wolfenstein, Parametrization of the Kobayashi-Maskawa Matrix. Phys. Rev. Lett. 51, 1945 (1983). https://doi.org/10.1103/ PhysRevLett.51.1945

8. D. Chakraverty, K. Huitu, A. Kundu, Effects of universal extra dimensions on $B^{0}-\bar{B}^{0}$ mixing. Phys. Lett. B 558, 173181 (2003). https://doi.org/10.1016/S0370-2693(03)00281-8. arXiv:hep-ph/0212047

9. A.J. Buras, M. Spranger, A. Weiler, The impact of universal extra dimensions on the unitarity triangle and rare $\mathrm{K}$ and $\mathrm{B}$ decays. Nucl. Phys. B 660, 225-268 (2003). https://doi.org/10. 1016/S0550-3213(03)00250-5. arXiv:hep-ph/0212143

10. S. Choudhury, N. Gaur, A. Goyal, N. Mahajan, $B_{d}-\bar{B}_{d}$ mass difference in little Higgs model. Phys. Lett. B 601, 164-170 (2004). https://doi.org/10.1016/j.physletb.2004.09.051. arXiv:hep-ph/0407050

11. W. Altmannshofer, A.J. Buras, D. Guadagnoli, The MFV limit of the MSSM for low tan(beta): Meson mixings revisited. JHEP 11, 065 (2007). https://doi.org/10.1088/1126-6708/2007/11/065. arXiv:hep-ph/0703200

12. M. Carlucci, P. Colangelo, F. De Fazio, Rare B(s) decays to eta and eta-prime final states. Phys. Rev. D 80, 055023 (2009). https://doi. org/10.1103/PhysRevD.80.055023. arXiv:0907.2160

13. Q.-Y. Hu, X.-Q. Li, Y.-D. Yang, M.-D. Zheng, $B_{s(d)}-\bar{B}_{s(d)}$ Mixing and $B_{s} \rightarrow \mu^{+} \mu^{-}$Decay in the NMSSM with the flavour expansion theorem. JHEP 06, 133 (2019). https://doi.org/10.1007/ JHEP06(2019)133. arXiv:1903.06927

14. T. Appelquist, H.-C. Cheng, B.A. Dobrescu, Bounds on universal extra dimensions. Phys. Rev. D 64, 035002 (2001). https://doi.org/ 10.1103/PhysRevD.64.035002. arXiv:hep-ph/0012100

15. A.J. Buras, F. Parodi, A. Stocchi, The CKM matrix and the unitarity triangle: Another look. JHEP 01, 029 (2003). https://doi.org/10. 1088/1126-6708/2003/01/029. arXiv:hep-ph/0207101

16. G. D'Ambrosio, G.F. Giudice, G. Isidori, A. Strumia, Minimal flavor violation: An Effective field theory approach. Nucl. Phys. B 645, 155-187 (2002). https://doi.org/10.1016/ S0550-3213(02)00836-2. arXiv:hep-ph/0207036

17. G. Servant, T.M.P. Tait, Elastic Scattering and Direct Detection of Kaluza-Klein Dark Matter. New J. Phys. 4, 99 (2002). https://doi. org/10.1088/1367-2630/4/1/399. arXiv:hep-ph/0209262

18. G. Servant, T.M.P. Tait, Is the lightest Kaluza-Klein particle a viable dark matter candidate? Nucl. Phys. B 650, 391419 (2003). https://doi.org/10.1016/S0550-3213(02)01012-X. arXiv:hep-ph/0206071

19. H.-C. Cheng, J.L. Feng, K.T. Matchev, Kaluza-Klein dark matter. Phys. Rev. Lett. 89, 211301 (2002). https://doi.org/10.1103/ PhysRevLett.89.211301. arXiv:hep-ph/0207125

20. D. Majumdar, Detection rates for Kaluza-Klein dark matter. Phys. Rev. D 67, 095010 (2003). https://doi.org/10.1103/PhysRevD.67. 095010. arXiv:hep-ph/0209277

21. F. Burnell, G.D. Kribs, The Abundance of Kaluza-Klein dark matter with coannihilation. Phys. Rev. D 73, 015001 (2006). https://doi. org/10.1103/PhysRevD.73.015001. arXiv:hep-ph/0509118

22. K. Kong, K.T. Matchev, Precise calculation of the relic density of Kaluza-Klein dark matter in universal extra dimensions. JHEP 01, 038 (2006). https://doi.org/10.1088/1126-6708/2006/01/038. arXiv:hep-ph/0509119

23. M. Kakizaki, S. Matsumoto, M. Senami, Relic abundance of dark matter in the minimal universal extra dimension model. Phys. Rev. D 74, 023504 (2006). https://doi.org/10.1103/PhysRevD.74. 023504. arXiv:hep-ph/0605280

24. G. Belanger, M. Kakizaki, A. Pukhov, Dark matter in UED: The Role of the second KK level. JCAP 1102, 009 (2011). https://doi. org/10.1088/1475-7516/2011/02/009. arXiv:1012.2577

25. K.R. Dienes, E. Dudas, T. Gherghetta, Extra space-time dimensions and unification. Phys. Lett. B 436, 55-65 (1998). https://doi.org/ 10.1016/S0370-2693(98)00977-0. arXiv:hep-ph/9803466

26. K.R. Dienes, E. Dudas, T. Gherghetta, Grand unification at intermediate mass scales through extra dimensions. Nucl. Phys. B 537, 47-108 (1999). https://doi.org/10.1016/S0550-3213(98)00669-5. arXiv:hep-ph/9806292

27. G. Bhattacharyya, A. Datta, S.K. Majee, A. Raychaudhuri, Power law blitzkrieg in universal extra dimension scenarios. Nucl. Phys. B 760, 117-127 (2007). https://doi.org/10.1016/j.nuclphysb.2006. 10.018. arXiv:hep-ph/0608208 
28. K. Hsieh, R.N. Mohapatra, S. Nasri, Dark matter in universal extra dimension models: Kaluza-Klein photon and right-handed neutrino admixture. Phys. Rev. D 74, 066004 (2006). https://doi.org/ 10.1103/PhysRevD.74.066004. arXiv:hep-ph/0604154

29. Y. Fujimoto, K. Nishiwaki, M. Sakamoto, R. Takahashi, Realization of lepton masses and mixing angles from point interactions in an extra dimension. JHEP 10, 191 (2014). https://doi.org/10.1007/ JHEP10(2014)191. arXiv:1405.5872

30. P.R. Archer, The Fermion Mass Hierarchy in Models with Warped Extra Dimensions and a Bulk Higgs. JHEP 09, 095 (2012). https:// doi.org/10.1007/JHEP09(2012)095. arXiv:1204.4730

31. H. Georgi, A.K. Grant, G. Hailu, Brane couplings from bulk loops. Phys. Lett. B 506, 207-214 (2001). https://doi.org/10.1016/ S0370-2693(01)00408-7. arXiv:hep-ph/0012379

32. H.-C. Cheng, K.T. Matchev, M. Schmaltz, Radiative corrections to Kaluza-Klein masses. Phys. Rev. D 66, 036005 (2002). https://doi. org/10.1103/PhysRevD.66.036005. arXiv:hep-ph/0204342

33. G.R. Dvali, G. Gabadadze, M. Kolanovic, F. Nitti, The Power of brane induced gravity. Phys. Rev. D 64, 084004 (2001). https://doi. org/10.1103/PhysRevD.64.084004. arXiv:hep-ph/0102216

34. M. Carena, T.M.P. Tait, C.E.M. Wagner, Branes and Orbifolds are Opaque. Acta Phys. Polon. B 33, 2355 (2002). arXiv:hep-ph/0207056

35. F. del Aguila, M. Perez-Victoria, J. Santiago, Bulk fields with general brane kinetic terms. JHEP 02, 051 (2003). https://doi.org/10. 1088/1126-6708/2003/02/051. arXiv:hep-th/0302023

36. F. del Aguila, M. Perez-Victoria, J. Santiago, Some consequences of Brane kinetic terms for bulk fermions. In 38th Rencontres de Moriond on Electroweak Interactions and Unified Theories Les Arcs, France, March 15-22, 2003 (2003). arXiv:hep-ph/0305119

37. F. del Aguila, M. Perez-Victoria, J. Santiago, Physics of brane kinetic terms. Acta Phys. Polon. B 34, 5511-5522 (2003). arXiv:hep-ph/0310353

38. C. Schwinn, Higgsless fermion masses and unitarity. Phys. Rev. D 69, 116005 (2004). https://doi.org/10.1103/PhysRevD.69.116005. arXiv:hep-ph/0402118

39. T. Flacke, A. Menon, D.J. Phalen, Non-minimal universal extra dimensions. Phys. Rev. D 79, 056009 (2009). https://doi.org/10. 1103/PhysRevD.79.056009. arXiv:0811.1598

40. A. Datta, U.K. Dey, A. Shaw, A. Raychaudhuri, Universal ExtraDimensional Models with Boundary Localized Kinetic Terms: Probing at the LHC. Phys. Rev. D 87, 076002 (2013). https://doi. org/10.1103/PhysRevD.87.076002 arXiv:1205.4334

41. T. Flacke, K. Kong, S.C. Park, Phenomenology of universal extra dimensions with bulk-masses and Brane-localized terms. JHEP 05, 111 (2013). https://doi.org/10.1007/JHEP05(2013)111. arXiv: 1303.0872

42. T. Flacke, K. Kong, S.C. Park, $126 \mathrm{GeV}$ Higgs in next-to-minimal universal extra dimensions. Phys. Lett. B 728, 262-267 (2014). https://doi.org/10.1016/j.physletb.2013.11.046. arXiv:1309.7077

43. J. Bonnevier, H. Melbeus, A. Merle, T. Ohlsson, Monoenergetic gamma-rays from non-minimal Kaluza-Klein dark matter annihilations. Phys. Rev. D 85, 043524 (2012). https://doi.org/10.1103/PhysRevD.85.109902 https://doi.org/10. 1103/PhysRevD.85.043524. arXiv:1104.1430

44. A. Datta, U.K. Dey, A. Raychaudhuri, A. Shaw, Boundary Localized Terms in Universal Extra-Dimensional Models through a Dark Matter perspective. Phys. Rev. D 88, 016011 (2013). https://doi. org/10.1103/PhysRevD.88.016011. arXiv:1305.4507

45. U.K. Dey, T.S. Ray, Constraining minimal and nonminimal universal extra dimension models with Higgs couplings. Phys. Rev. D 88, 056016 (2013). https://doi.org/10.1103/PhysRevD.88.056016. arXiv: 1305.1016

46. A. Datta, K. Nishiwaki, S. Niyogi, Non-minimal universal extra dimensions: the strongly interacting sector at the Large
Hadron Collider. JHEP 11, 154 (2012). https://doi.org/10.1007/ JHEP11(2012)154. arXiv:1206.3987

47. A. Datta, K. Nishiwaki, S. Niyogi, Non-minimal Universal Extra Dimensions with Brane Local Terms: The Top Quark Sector. JHEP 01, 104 (2014). https://doi.org/10.1007/JHEP01(2014)104. arXiv: 1310.6994

48. A. Datta, A. Raychaudhuri, A. Shaw, LHC limits on KK-parity non-conservation in the strong sector of universal extra-dimension models. Phys. Lett. B 730, $42-49$ (2014). https://doi.org/10.1016/ j.physletb.2014.01.027. arXiv:1310.2021

49. A. Shaw, KK-parity non-conservation in UED confronts LHC data. Eur. Phys. J. C 75, 33 (2015). https://doi.org/10.1140/epjc/ s10052-014-3245-0. arXiv:1405.3139

50. A. Shaw, Status of exclusion limits of the KK-parity nonconserving resonance production with updated $13 \mathrm{TeV}$ LHC. Acta Phys. Polon. B 49, 1421 (2018). https://doi.org/10.5506/ APhysPolB.49.1421. arXiv:1709.08077

51. N. Ganguly, A. Datta, Exploring non minimal universal extra dimensional model at the LHC. JHEP 10, 072 (2018). https://doi. org/10.1007/JHEP10(2018)072. arXiv:1808.08801

52. T. Jha, A. Datta, $Z \rightarrow b \bar{b}$ in non-minimal Universal Extra Dimensional Model. JHEP 03, 012 (2015). https://doi.org/10. 1007/JHEP03(2015)012. arXiv: 1410.5098

53. A. Datta, A. Shaw, Nonminimal universal extra dimensional model confronts $\mathrm{B}_{s} \rightarrow \mu^{+} \mu^{-}$. Phys. Rev. D 93, 055048 (2016). https:// doi.org/10.1103/PhysRevD.93.055048. arXiv:1506.08024

54. A. Datta, A. Shaw, Effects of non-minimal Universal Extra Dimension on $B \rightarrow X_{s} \gamma$. Phys. Rev. D 95, 015033 (2017). https://doi. org/10.1103/PhysRevD.95.015033. arXiv:1610.09924

55. A. Shaw, Looking for $B \rightarrow X_{S} \ell^{+} \ell^{-}$in a nonminimal universal extra dimensional model. Phys. Rev. D 99, 115030 (2019). https:// doi.org/10.1103/PhysRevD.99.115030. arXiv:1903.10302

56. A. Biswas, A. Shaw, S.K. Patra, $\mathcal{R}\left(D^{(*)}\right)$ anomalies in light of a nonminimal universal extra dimension. Phys. Rev. D 97, 035019 (2018). https://doi.org/10.1103/PhysRevD.97.035019. arXiv: 1708.08938

57. S. Dasgupta, U.K. Dey, T. Jha, T.S. Ray, Status of a flavormaximal nonminimal universal extra dimension model. Phys. Rev. D 98, 055006 (2018). https://doi.org/10.1103/PhysRevD.98. 055006. arXiv:1801.09722

58. J.-P. Lee, $B$ anomalies in the nonminimal universal extra dimension model. Phys. Rev. D 100, 075005 (2019). https://doi.org/10.1103/ PhysRevD.100.075005. arXiv:1906.07345

59. U.K. Dey, T. Jha, Rare top decays in minimal and nonminimal universal extra dimension models. Phys. Rev. D 94, 056011 (2016). https://doi.org/10.1103/PhysRevD.94.056011. arXiv:1602.03286

60. C.-W. Chiang, U.K. Dey, T. Jha, $t \rightarrow c g$ and $t \rightarrow c Z$ in universal extra-dimensional models. Eur. Phys. J. Plus 134, 210 (2019). https://doi.org/10.1140/epjp/i2019-12607-1. arXiv:1807.01481

61. T. Jha, Unitarity Constraints on non-minimal Universal Extra Dimensional Model. J. Phys. G 45, 115002 (2018). https://doi. org/10.1088/1361-6471/aade80. arXiv:1604.02481

62. Particle Data Group collaboration, P. Zyla et al., Review of particle physics. PTEP2020, 083C01 (2020). https://doi.org/10. 1093/ptep/ptaa104

63. The CKMfitter Group, Updated results on the CKM matrix, including results presented up to summer 19, [The CKMfitter Group, 15 December, 2019] (2019). https://ckmfitter.in2p3.fr/www/results/ plots_summer19/num/ckmEval_results_summer19.pdf

64. A. Datta, A. Shaw, A note on gauge-fixing in the electroweak sector of non-minimal UED. Mod. Phys. Lett. A 31, 1650181 (2016). https://doi.org/10.1142/S0217732316501819. arXiv:1408.0635

65. A.J. Buras, M. Jamin, P.H. Weisz, Leading and Next-to-leading QCD Corrections to $\epsilon$ Parameter and $B^{0}-\bar{B}^{0}$ Mixing in the Presence of a Heavy Top Quark. Nucl. Phys. B 347, 491-536 (1990). https://doi.org/10.1016/0550-3213(90)90373-L 
66. J. Urban, F. Krauss, U. Jentschura, G. Soff, Next-to-leading order QCD corrections for the $B^{0}-\bar{B}^{0}$ mixing with an extended Higgs sector. Nucl. Phys. B 523, 40-58 (1998). https://doi.org/10.1016/ S0550-3213(98)00043-1. arXiv:hep-ph/9710245

67. A.J. Buras, M.E. Lautenbacher, G. Ostermaier, Waiting for the top quark mass, $K^{+} \rightarrow \pi^{+} \nu \bar{\nu} B_{s}^{0}-\overline{B_{s}^{0}}$ mixing and $\mathrm{CP}$ asymmetries in B decays. Phys. Rev. D 50, 3433-3446 (1994). https://doi.org/ 10.1103/PhysRevD.50.3433. arXiv:hep-ph/9403384

68. A.J. Buras, Weak Hamiltonian, CP violation and rare decays. In Les Houches Summer School in Theoretical Physics, Session 68: Probing the Standard Model of Particle Interactions, vol. 6 (1998), pp. 281-539. arXiv:hep-ph/9806471

69. A.J. Buras, Flavor dynamics: $\mathrm{CP}$ violation and rare decays. Subnucl. Ser. 38, 200-337 (2002). https://doi.org/10.1142/ 9789812778253_0005. arXiv:hep-ph/0101336

70. HFLAV collaboration, Y.S. Amhis et al., Averages of $b$ hadron, $c$-hadron, and $\tau$-lepton properties as of 2018 (2019). arXiv:1909.12524

71. A. Lenz, G. Tetlalmatzi-Xolocotzi, Model-independent bounds on new physics effects in non-leptonic tree-level decays of B-mesons. JHEP 07, 177 (2020). https://doi.org/10.1007/JHEP07(2020)177. arXiv: 1912.07621

72. A. Banerjee, G. Bhattacharyya, N. Kumar, Impact of Yukawa-like dimension-five operators on the Georgi-Machacek model. Phys. Rev. D 99, 035028 (2019). https://doi.org/10.1103/PhysRevD.99. 035028. arXiv: 1901.01725

73. A. Datta, S. Raychaudhuri, Vacuum Stability Constraints and LHC Searches for a Model with a Universal Extra Dimension. Phys. Rev. D 87, 035018 (2013). https://doi.org/10.1103/PhysRevD.87. 035018. arXiv: 1207.0476

74. P. Dey, G. Bhattacharyya, A Comparison of ultraviolet sensitivities in universal, nonuniversal, and split extra dimensional models. Phys. Rev. D 70, 116012 (2004). https://doi.org/10.1103/ PhysRevD.70.116012. arXiv:hep-ph/0407314

75. BABAR collaboration, J.P. Lees et al., Measurement of the $B \rightarrow$ $X_{s} l^{+} l^{-}$branching fraction and search for direct CP violation from a sum of exclusive final states. Phys. Rev. Lett. 112, 211802 (2014). https://doi.org/10.1103/PhysRevLett.112.211802. arXiv: 1312.5364

76. P. Nath, M. Yamaguchi, Effects of Kaluza-Klein excitations on $(g-2)_{\mu}$. Phys. Rev. D 60, 116006 (1999). https://doi.org/10.1103/ PhysRevD.60.116006. arXiv:hep-ph/9903298

77. T. Appelquist, H.-U. Yee, Universal extra dimensions and the Higgs boson mass. Phys. Rev. D 67, 055002 (2003). https://doi.org/10. 1103/PhysRevD.67.055002. arXiv:hep-ph/0211023

78. A.J. Buras, A. Poschenrieder, M. Spranger, A. Weiler, The Impact of universal extra dimensions on $B \rightarrow X_{s} \gamma, B \rightarrow$ $X_{s}$ gluon, $B \rightarrow X_{s} \mu^{+} \mu^{-}, K_{L} \rightarrow \pi^{0} e^{+} e^{-}$and $\epsilon^{\prime} / \epsilon$. Nucl. Phys. B 678, 455-490 (2004). https://doi.org/10.1016/j.nuclphysb.2003. 11.010. arXiv:hep-ph/0306158
79. K. Agashe, N.G. Deshpande, G.H. Wu, Universal extra dimensions and $b \rightarrow s \gamma$. Phys. Lett. B 514, 309-314 (2001). https://doi.org/ 10.1016/S0370-2693(01)00791-2. arXiv:hep-ph/0105084

80. J.F. Oliver, J. Papavassiliou, A. Santamaria, Universal extra dimensions and $Z \rightarrow b \bar{b}$. Phys. Rev. D 67, 056002 (2003). https://doi. org/10.1103/PhysRevD.67.056002. arXiv:hep-ph/0212391

81. A. Strumia, Bounds on Kaluza-Klein excitations of the SM vector bosons from electroweak tests. Phys. Lett. B 466, 107114 (1999). https://doi.org/10.1016/S0370-2693(99)01102-8. arXiv:hep-ph/9906266

82. T.G. Rizzo, J.D. Wells, Electroweak precision measurements and collider probes of the standard model with large extra dimensions. Phys. Rev. D 61, 016007 (2000). https://doi.org/10.1103/ PhysRevD.61.016007. arXiv:hep-ph/9906234

83. C.D. Carone, Electroweak constraints on extended models with extra dimensions. Phys. Rev. D 61, 015008 (2000). https://doi.org/ 10.1103/PhysRevD.61.015008. arXiv:hep-ph/9907362

84. A. Belyaev, M. Brown, J. Moreno, C. Papineau, Discovering Minimal Universal Extra Dimensions (MUED) at the LHC. JHEP 06, 080 (2013). https://doi.org/10.1007/JHEP06(2013)080. arXiv: 1212.4858

85. T. Golling et al., Physics at a $100 \mathrm{TeV}$ pp collider: beyond the standard model phenomena. InCERN Yellow Report (2017), pp. 441-634. https://doi.org/10.23731/CYRM-2017-003. 441. arXiv: 1606.00947

86. Y. Gershtein et al., Working Group Report: new particles, forces, and dimensions. In Proceedings, 2013 Community Summer Study on the Future of U.S. Particle Physics: Snowmass on the Mississippi (CSS2013): Minneapolis, MN, USA, July 29-August 6, 2013 (2013). arXiv:1311.0299. http://lss.fnal.gov/archive/2013/ conf/fermilab-conf-13-584-t.pdf

87. D. Choudhury, K. Ghosh, Bounds on universal extra dimension from LHC Run I and II data. Phys. Lett. B 763, 155-160 (2016). https://doi.org/10.1016/j.physletb.2016.10.010. arXiv:1606.04084

88. J. Beuria, A. Datta, D. Debnath, K.T. Matchev, LHC Collider phenomenology of minimal universal extra dimensions. Comput. Phys. Commun. 226, 187-205 (2018). https://doi.org/10.1016/j. cpc.2017.12.021. arXiv:1702.00413

89. S. Chakraborty, S. Niyogi, K. Sridhar, Constraining compressed versions of MUED and MSSM using soft tracks at the LHC. JHEP 07, 105 (2017). https://doi.org/10.1007/JHEP07(2017)105. arXiv: 1704.07048

90. N. Deutschmann, T. Flacke, J.S. Kim, Current LHC constraints on minimal universal extra dimensions. Phys. Lett. B 771, 515-520 (2017). https://doi.org/10.1016/j.physletb.2017.06.004. arXiv: 1702.00410 